\title{
Selberg Super-Trace Formula for Super Riemann Surfaces II: Elliptic and Parabolic Conjugacy Classes, and Selberg Super-Zeta Functions
}

\section{Grosche}

The Blackett Laboratory, Imperial College of Science, Technology and Medicine, Prince Consort Road, London SW7 2BZ, UK*

Received September 10, 1991; in revised form June 12, 1992

\begin{abstract}
Further contributions developing a super analogue of the classical Selberg trace formula, the Selberg super-trace formula, are presented. This paper deals with the calculation of contributions arising from elliptic and parabolic conjugacy classes to the Selberg super-trace formula for super Riemann surfaces. Analytic properties and the functional equation for the corresponding Selberg super-zeta function $R_{0}, R_{1}$ and $Z_{S}$, respectively, are derived and discussed. In particular, the elliptic contributions to a super Fuchsian group only alter the multiplicities of the "trivial" zeros and poles of the Selberg super-zeta function $R_{0}$, $R_{1}$ and $Z_{S}$, respectively, already due to the hyperbolic conjugacy classes. The parabolic conjugacy classes introduce new features in the analytical structure.
\end{abstract}

\section{Introduction}

In this paper I want to present further contributions to develop a super analogue to the classical Selberg trace formula. This includes also an investigation of the analytic properties of the Selberg super-zeta functions.

Trace formulae emerge in various fields of mathematics and physics, in the latter particularly in the study of fundamental forces. The original version of the Selberg trace formula by Selberg [55] has come from the intention to study number theoretical problems. Actually the Selberg trace formula has some striking similarities with the Weil formula [66] and there is in fact a close relation between the areas of analytic number theory, eigenvalues on compact Riemann surfaces and the Selberg trace formula (e.g. [33]). Of particular interest in all these studies are the analytic properties (zeros and poles) of the Selberg zeta-function $Z(s)$.

There are other reasons as well for studying trace formulae in particular in physics. The Selberg trace formula is a special case of a general class of trace

* Present Address: International School for Advanced Studies, SISSA, Via Beirut 4, 34014 Trieste, Miramare, Italy 
formulae connecting classical mechanics and quantum theory. Quite typically, trace formulae for quantum systems emerge in the semiclassical approximation of the Feynman kernel (propagator) as systematically developed by Gutzwiller [30] (see also Littlejohn [37], and Sieber and Steiner [56] and references therein). In particular in the study of quantum chaos, the arising dynamical zeta-functions, where the Selberg zeta-function is just one, serves as an important tool in the "Quantization of Chaos," see e.g. Refs. [4, 44, 56].

The Selberg trace formula is Gutzwiller's trace formula on closed Riemann surfaces. In its simplest form the Selberg trace formula reads $[33,34,55]$ ( $p_{n}$ is defined by $E_{n}=p_{n}^{2}+\frac{1}{4}$ with $E_{n}$ the eigenvalues of the non-euclidean Laplacian on the Riemann surface, respectively, on the fundamental domain $\mathscr{F}$ )

$$
\sum_{n} h\left(p_{n}\right)=\frac{A(\mathscr{F})}{4 \pi} \int_{-\infty}^{\infty} p h(p) \tanh \pi p d p+\sum_{\gamma} \sum_{k=1}^{\infty} \frac{l_{\gamma} g\left(k l_{\gamma}\right)}{2 \sinh \frac{k l_{\gamma}}{2}} .
$$

$A(\mathscr{F})$ denotes the (non-euclidean) area of $\mathscr{F}$ (in the actual case $A=4 \pi(g-1)$ with $g$ the genus of the Riemann surface), and the summation of the classification $\gamma$ is a summation over distinct hyperbolic conjugacy classes, tesselating the hyperbolic plane $\mathscr{H}$ (Pioncaré upper half-plane, Poincaré disc, Lobaschevsky plane) $\mathscr{H}=\{(x, y) \mid x \in \mathbf{R}, y>0\}$, endowed with the hyperbolic geometry $d s^{2}=\left(d x^{2}+d y^{2}\right) / y^{2}$, by fundamental domains $\mathscr{F}$ of $\Gamma \cdot \Gamma / \mathscr{H}$ then actually is a Riemann surface defined by the action of $\Gamma$ on $\mathscr{H}$. Equation (1.1) is an exact formula, as rediscovered by Gutzwiller [31] by considering the trace of the heat kernel (Feynman path integral), respectively the resolvent kernel for the free motion of the entire Poincaré upper half-plane $[26,28]$.

The trace formula (1.1) can be used to analyse classical and quantum motion on closed Riemann surfaces, e.g. Ref. [67], the Hadamard-Gutzwiller model [3], and Artin's billiard [44], respectively. These models are studied in the context of the question of quantum chaos on hyperbolic spaces (Balazs and Voros [5]). Furthermore, Eq. (1.1) can be used to calculate determinants of Maass Laplacians $\Delta_{k}^{( \pm)}=-y^{2}\left(\partial_{x}^{2}+\partial_{y}^{2}\right)+i k y \partial_{x}+k(k \pm 1)$ on Riemann surfaces, see Bolte and Steiner [12], D'Hoker and Phong [16, 18], and Takhtajan and Zograf [59], i.e. the latter $\operatorname{define} \operatorname{det}\left(\Delta_{k}^{ \pm}\right) \propto Z^{\prime}(1)$ for $k=0,1$, and $\operatorname{det}\left(\Delta_{k}^{ \pm}\right) \propto Z(k)$ otherwise, where $Z(s)$ is the Selberg zeta function.

Clearly, the trace formula (1.1) can be generalized to automorphic $k$-forms [34], to higher dimensional hyperbolic spaces and generalized Lorentz groups, see Arthur [2], Subia [58] and Venkov [61, 62], respectively. Also bordered Riemann surfaces can be included, see e.g. Ref. [67], Bolte and Steiner [13] and Venkov [62], and automorphic potentials as well [65]. Further, Selberg trace formulae can be used to determine corrections to Weyl's law on Riemann surfaces [4, 63, 64], and to study the analytic properties of the Selberg zeta function (Hejhal [34], McKean [38], Selberg [55], Steiner [57] and Venkov [63, 64]).

Whereas the study of Laplacians on Riemann surfaces and their determinants is, of course, of important mathematical interest, the full power of the formalism in the study of fundamental forces is revealed in the Polyakov approach to string theory, see D'Hoker and Phong [15, 16], Green, Schwarz and Wittern [25] and Polyakov $[49,50]$. The convergence properties of the perturbation expansion can be analysed by the growing properties of the Selberg zeta function with respect to the genus $g$ [29], and actually the perturbation expansion is highly divergent and 
not Borel summable. Of course, there is not only a bosonic string theory but there is a fermionic, supersymmetric, heterotic and adelic version, too. It is therefore evident that corresponding trace formulae and a theory of harmonic analysis on corresponding hyperbolic spaces (super,... .) should be developed to deal with fermionic strings (D'Hoker and Phong [17], Gliozzi, Scherk and Olive [20], Green and Schwarz [23], Moore, Nelson and Polchinski [45], Neveu and Schwarz [46], Polyakov [50] and Ramond [52]), respectively superstrings, see Green and Schwarz [22, 24, 54].

Quite analogously as in the bosonic case, the considerations of Fuchsian groups, a Selberg trace formula, Selberg zeta functions and determinants of Laplacians expressed by Selberg zeta functions, can be incorporated into the theory of supersymmetry (Aoki [1], Baranov et al. [6-9], and [27]). However, as started by the work of Baranov et al. [6,7] and more thoroughly studied in [27], the first version of the super analogue of the Selberg trace formula was developed only for hyperbolic conjugacy classes of super Fuchsian groups. As is wellknown, a complete study of the Selberg trace formula (and its generalizations) must include elliptic and parabolic conjugacy classes, too. In particular, the parabolic conjugacy classes in general give rise to a continuous spectrum of the relevant Laplacians on the corresponding fundamental domains $\mathscr{F}$. From the Polyakov point of view in string theory, the incorporation of elliptic and parabolic conjugacy classes correspond to punctured Riemann surfaces, where vertex operators are attached, and cusps at infinity ("in" and "out-popping" strings), respectively, and therefore should provide a more comprehensive approach.

In a previous publication [27] I have completed the study of the Selberg super-trace formula as introduced by Baranov et al. [6-9]. However, only hyperbolic conjugacy classes were taken into account. The main objective of this paper will be the incorporation of elliptic and parabolic conjugacy classes into the super analogue of the Selberg trace formula. Whereas the case of the elliptic conjugacy classes is not too difficult to handle, the parabolic conjugacy classes must be treated carefully due to the arising continuous spectrum of the Dirac-Laplace operator $\square$, and the Selberg super-trace formula must be regularized by subtracting this contribution. I am only concerned with the developing of the Selberg super-trace formula, respectively the investigation of the analytic properties of the Selberg super-zeta functions. My motivation is therefore to develop a superanalogue as close as possible to the classical Selberg trace formula. I will rely heavily on the classical reports on the subject of the theory of automorphic forms, i.e. Hejhal [34] and Venkov [64]. Insofar the discussions remain formal with no direct application to particular physical situations, say in string theory. Possible applications are the evaluation of super-determinants of Laplace-Dirac operators on super-Riemann surfaces, a $N(E)$ staircase analysis (Weyl's law), and a $N(l)$ staircase analysis (Huber's law), respectively. Whereas in the former case the explicit calculation seems quite involved with no obvious solution (see however Takhtajan and Zograf [59] as mentioned above) and deserves further study, an investigation of the latter problems will be given separately.

In the next section, a short review will be given of the relevant notation and description of the Poincaré super upper half-plane $\mathscr{H}^{(1 \mid 1)}=$ $\left\{Z=(z, \theta) \in \mathbf{C}^{(1 \mid 1)} \mid \mathfrak{J}(z)>0\right\}$. This includes the definition of the group action of super Fuchsian groups $\Gamma$, properties of $\square$ and the definition of the Selberg super operator $L$ on $\mathscr{H}^{(1 \mid 1)}$, respectively, and the statement of the Selberg super-trace formula for hyperbolic conjugacy classes. These topics were studied and described 
in some length in [27], so that our discussion here is only sketchy. In the third and fourth sections, respectively, the contributions from the elliptic and parabolic conjugacy classes will be calculated. In the fifth section I analyse the Selberg super-zeta functions $R_{0}, R_{1}$ and $Z_{S}$, respectively (where the last one can be written as a combination of the former two), with respect to the incorporation of these new contributions. The sixth section contains a summary and discussion of the results.

\section{Super Riemann Surfaces and the Selberg Super-Trace Formula for Hyperbolic Conjugacy Classes}

We sketch some important facts about super Riemann surfaces. For more details I refer to Batchelor et al. [10,11], DeWitt [14], Moore, Nelson and Polchinski [45], Ninnemann [47], Rabin and Crane [51], and Rogers [53]. Let us start with a $(1,1)$ (complex)-dimensional (not necessarily) flat superspace, parameterized by even coordinates $z \in \mathbf{C}_{c}$ and odd (Grassmann) coordinates $\theta \in \mathbf{C}_{a}$, respectively. Let $\Lambda_{\infty}$ be the infinite dimensional vector space generated by elements $\zeta_{a}(a=1,2, \ldots)$ with basis $1, \zeta_{a}, \zeta_{a} \zeta_{b}, \ldots(a<b)$ and the anticommuting relation $\zeta_{a} \zeta_{b}=-\zeta_{b} \zeta_{a}, \forall_{a, b}$. Every $z \in \Lambda_{\infty}$ can be decomposed as $z=z_{B}+z_{S}$ with $z_{B} \in$ $\mathbf{C}_{c} \equiv \mathbf{C}, z_{S}=\sum_{n} \frac{1}{n !} c_{a_{1}} \ldots, a_{n} \zeta^{a_{n}} \ldots \zeta^{a_{1}}$, with the $c_{a_{1}, \ldots, a_{n}} \in \mathbf{C}_{a}$ totally antisymmetric. $z_{B}$ and $z_{S}$, respectively, are called the body and soul of the supernumber $z$, respectively. In the fermionic string theory one is interested in super conformal symmetry. The notion of superspace and supermanifolds $[11,14,51,53]$ enables one to represent these symmetry transformations as pure geometric transformations in the coordinates $Z=(z, \theta) \in \mathbf{C}_{c} \times \mathbf{C}_{a}$. Let us consider the operator $D=\theta$ $\partial_{z}+\partial_{\theta}$ (note $D^{2}=\partial_{z}$ ). Further we consider a general superanalytic coordinate transformation $\tilde{z}=\tilde{z}(z, \theta), \tilde{\theta}=\tilde{\theta}(z, \theta)$. A superanalytic coordinate transformation is called superconformal, iff the $(0,1)$-dimensional subspace of the tangential space generated by the action of $D$ is invariant under such a coordinate transformation, i.e. $D=(D \tilde{\theta}) \tilde{D}$. Due to a theorem of Batchelor [10] every differentiable supermanifold is split, and in particular every complex supermanifold of dimension $(d \mid 1)$. The super Riemann surfaces in question can be seen as a complex $(1,1)$-dimensional supermanifold, respectively a real (2|2)-dimensional manifold, where the coordinate transformations are super conformal mappings. In the case of non-euclidean harmonic analysis in the context of super Riemann surfaces we consider the group $\operatorname{OSp}(2, \mathbf{C})$ of super conformal automorphisms on super Riemann surfaces as a natural generalization of Möbius transformations. They have the form

$$
\begin{array}{r}
\operatorname{OSp}\left(2,1 ; \mathbf{C}_{c}^{2} \times \mathbf{C}_{a}\right):=\left\{\gamma=\left(\begin{array}{ccc}
a & b & \chi_{\gamma}(b \alpha-\alpha \beta) \\
c & d & \chi_{\gamma}(d \alpha-c \beta) \\
\alpha & \beta & \chi_{\gamma}(1-\alpha \beta)
\end{array}\right) \mid a, b, c, d \in \mathbf{C}_{c} ;\right. \\
\left.\alpha, \beta \in \mathbf{C}_{a} ; a d-b c=1+\alpha \beta ; \operatorname{sdet} \gamma=\chi_{\gamma}= \pm 1\right\},
\end{array}
$$

together with the complex conjugate rules

$$
\overline{f+g}=\bar{f}+\bar{g}, \quad \overline{f \cdot g}=\bar{g} \cdot \bar{f} .
$$


Its generators are the operators $L_{0}, L_{1}, L_{-1}, G_{\frac{1}{2}}$ and $G_{-\frac{1}{2}}$ of the Neveu-Schwarz sector of the super Virasoro algebra of the fermionic string. Elements $\gamma \in \operatorname{OSp}\left(2,1 ; \mathbf{C}_{c}^{2} \times \mathbf{C}_{a}\right)$ act on elements $x=\left(z_{1}, z_{2}, \xi\right) \in \mathbf{C}_{c}^{2} \times \mathbf{C}_{a} \backslash\{0\}$ by matrix multiplication $x^{\prime}=\gamma x$. By means of a local coordinate system $(z, \theta)=\left(z_{1} / z_{2}, \xi / z_{2}\right)$ and the requirements of superconformal transformation the super Möbius transformation has the form $[7,27,47,51,60]$

$$
z^{\prime}=\frac{a z+b}{c z+d}+\theta \frac{\alpha z+\beta}{(a z+d)^{2}}, \quad \theta^{\prime}=\frac{\alpha+\beta z}{c z+d}+\frac{\chi_{\gamma} \theta}{c z+d} .
$$

Due to the separability of the super Riemann surfaces in question, i.e. that it is split, the odd quantities $\alpha, \beta$ are not necessary and can be omitted. It is sufficient to consider transformations $\gamma \in \operatorname{OSp}(2,1)$ with $\alpha=\beta=0$ and the characters $\chi_{\gamma}$ which describe a spin structure. Furthermore $\gamma$ and $-\gamma$ describe the same transformation. We thus have that the automorphisms on $\mathscr{H}^{(1 \mid 1)}$ are given by

$$
\text { Aut } \mathscr{H}^{(1 \mid 1)}=\frac{\operatorname{OSp}(2 \mid 1, \mathbf{R})}{\{ \pm 1\}}
$$

and a super Fuchsian group $\Gamma$ denotes a discrete subgroup of Aut $\mathscr{H}^{(1 \mid 1)}$. Therefore we obtain for the transformations $z \rightarrow z^{\prime}$ and $\theta \rightarrow \theta^{\prime}$ [47],

$$
z^{\prime}=\frac{a z+b}{c z+d}, \quad \theta^{\prime}=\frac{\chi_{\gamma} \theta}{c z+d} .
$$

Let us introduce the quantities $N_{\gamma}$ and $\chi_{\gamma}$, respectively, by

$$
2 \cosh \frac{l_{\gamma}}{2}=N_{\gamma}^{\frac{1}{\gamma}}+N_{\gamma}^{-\frac{1}{2}}=a+d+\chi_{\gamma} \alpha \beta .
$$

$N_{\gamma}$ is called norm of an hyperbolic $\gamma \in \Gamma$ and $N_{\gamma_{0}}$ will denote the norm of a primitive parabolic $\gamma \in \Gamma$ and $l_{\gamma}=\ln N_{\gamma_{0}}$ the length corresponding to a primitive $\gamma_{0}$. As can be shown [41], Huber's law for the number of geodesics is valid, i.e. the number of geodesics of length $l$ is growing according to $N_{l}=\#$ (geodesics $\left.\leqq l\right) \propto e^{l} / l$. For $\operatorname{OSp}(2, \mathbf{R}) /\{ \pm 1\}$ a hyperbolic transformation is always conjugate to the transformation

$$
z^{\prime}=N_{\gamma} z, \quad \theta^{\prime}=\chi_{\gamma} \sqrt{N_{\gamma}} \theta,
$$

or in matrix representation

$$
\text { hyperbolic } \gamma \in \Gamma \text { conjugate to }\left(\begin{array}{ccc}
N_{\gamma}^{\frac{1}{\gamma}} & 0 & 0 \\
0 & N_{\gamma}^{-\frac{1}{2}} & 0 \\
0 & 0 & \chi_{\gamma}
\end{array}\right) \text {. }
$$

The generators of a particular super Fuchsian group of a super Riemann surface with genus $g$ obey the constraint

$$
\left(\gamma_{0} \gamma_{1}^{-1} \ldots \gamma_{2 g-2} \gamma_{2 g-1}^{-1}\right)\left(\gamma_{0}^{-1} \gamma_{1} \ldots \gamma_{2 g-2}^{-1} \gamma_{2 g-1}\right)=\mathbf{1}_{2 \mid 1} .
$$


In order to construct explicitly a metric on $\mathscr{H}^{(1 \mid 1)}[27,60]$ one starts with the super Vierbeins $\hat{E}_{M}{ }^{A}$ in flat superspace and performs a super Weyl transformation [35]. The scalar product has the form

$$
\left(\Phi_{1}, \Phi_{2}\right)=\int d V(Z) \Phi_{1}(Z) \bar{\Phi}_{2}(Z), \quad d V(Z)=\frac{d z d \bar{z} d \theta d \bar{\theta}}{2 Y}
$$

$(Y=y+\theta \bar{\theta}, y=\mathfrak{I}(z))$ for super functions $\Phi_{1}, \Phi_{2} \in L^{2}\left(\mathscr{H}^{(1 \mid 1)}\right)$. Two point-pair invariants are given by [41]

$$
\begin{aligned}
R(Z, W)= & \frac{|z-w-\theta v|^{2}}{Y V} \\
r(Z, W)= & \frac{2 \theta \bar{\theta}+i(v-i \bar{v})(\theta+i \bar{\theta})}{4 Y}+\frac{2 v \bar{v}+i(\theta-i \bar{\theta})(v+i \bar{v})}{4 V} \\
& +\frac{(v+i \bar{v})(\theta+i \bar{\theta}) \mathfrak{R}(z-w-\theta v)}{4 Y V}
\end{aligned}
$$

and are even and odd, respectively $\left(W=\left(u+i v, v_{1}+v_{2}\right), V=v+v \bar{v} / 2\right)$. We introduce the Dirac-Laplace operators $\square_{m}$ and $\hat{\square}_{m}$, respectively $[1,7]$

$$
\square_{m}=2 Y D \bar{D}-m(i \theta-\bar{\theta}) \bar{D}, \quad \hat{\square}_{m}=2 Y D \bar{D}+\frac{m}{2}(i \theta-\bar{\theta})(\bar{D}+i D),
$$

and $\square_{m}$ and $\hat{\square}_{m}$ are related by a linear isomorphism $\square_{m}=Y^{\frac{m}{2}}\left(\hat{\square}_{m}+\frac{m}{2}\right) Y^{-\frac{m}{2}}$. With the notation $-\Delta_{m}=-4 y^{2} \partial_{z} \partial_{z}+i m y \partial_{x}=-y^{2}\left(\partial_{x}^{2}+\partial_{y}^{2}\right)+i m y \partial_{x}$ we obtain for an even super function

$$
\Phi(z, \bar{z}, \theta, \bar{\theta})=A(z, \bar{z})+\frac{\theta \bar{\theta}}{y} B(z, \bar{z})
$$

the following equivalence

$$
\hat{\square}_{m} \Phi(z, \bar{z}, \theta, \bar{\theta})=s \Phi(z, \bar{z}, \theta, \bar{\theta}) \equiv\left\{\begin{array}{l}
-\Delta_{m} A(z, \bar{z})=s(1-s) A(z, \bar{z}), \\
B(z, \bar{z})=\frac{s}{2} A(z, \bar{z}) .
\end{array}\right.
$$

Therefore an explicit solution of Eq. (2.14) is given by [43]

$$
\Phi_{p, k}(z, \bar{z}, \theta, \bar{\theta})=\sqrt{\frac{2 i \sinh \pi p}{\pi^{3}}}\left(1+\frac{1+2 i p}{4 y} \theta \bar{\theta}\right) \sqrt{y} e^{i k x} K_{i p}(|k| y)
$$

with $s=\frac{1}{2}+i p$.

For convenience, let us cite the classical Selberg trace formula which we want to generalize to the super case. We follow Hejhal [34, Vol. II] and Venkov [64]. Let $\kappa$ be the number of inequivalent cusps $z_{j}(j=1, \ldots, \kappa)$. For each $z_{j}$ we corrsider the maximal subgroup $\Gamma_{j} \subset \Gamma$ which stabilizes it. The subgroup $\Gamma_{j}$ is generated by a single parabolic element $S_{j}$. For each $j=1, \ldots, \kappa$ there exists a transformation $g_{j} \in \operatorname{PSL}(2, \mathbf{R})$ such that $g_{j} \infty=z_{j}, g_{j}^{-1} S_{j} g_{j} z=S_{\infty} z=z+1(z \in \mathscr{H})$. Let $V$ be an $h$-dimensional complex vector space, $V=\mathbf{C}^{h}$. Let $U$ be an representation of 
$\Gamma$ which acts in the space $V$ and is unitary with respect to the inner product in $V$. For each $j=1, \ldots, \kappa$ we have a subspace $V_{j} \subset V$ of the operator $U\left(S_{j}\right)$, i.e. $V_{j}=\left\{v \in V \mid U\left(s_{j}\right) v=v\right\}$. Let $k_{j}=\operatorname{dim} V_{j}$ and $\kappa_{0}=\sum_{j=1}^{\kappa} k_{j} . k_{j}$ denotes the degree of singularity of the representation $U$ relative to the generator $S_{j}$ of $\Gamma_{j} \subset \Gamma$ and $\kappa_{0}$ denotes the degree of singularity of $\Gamma$ relative to $U$. For each $\alpha(\alpha=1, \ldots, \kappa)$ one chooses a basis $v_{1}(\alpha), \ldots, v_{h}(\alpha)$ for $V$ together with the operator

$$
U\left(S_{\alpha}\right)\left(1_{V}-P_{\alpha}\right) v_{l}(\alpha)=v_{l \alpha} v_{l}(\alpha),
$$

with $P_{\alpha}$ the projector on the subspace $V_{\alpha}$, and where it is supposed that we have the alternatives

$$
v_{l \alpha} v_{l}(\alpha)=\left\{\begin{array}{l}
0 \\
e^{2 \pi i \theta_{l \alpha}}
\end{array},\right.
$$

with the numbers $0<\theta_{l \alpha}<1$. For $\kappa_{0} \geqq 1$ the contributions corresponding to the parabolic conjugacy classes must be regularized, i.e. the continuous spectrum of the automorphic kernel must be subtracted from the Selberg trace formula. In order to do this one considers the Eisenstein series

$$
e(z, s, \alpha, v, \Gamma, U)=e(z, s, \alpha, v)=\sum_{\gamma \in \Gamma_{\alpha} \backslash \Gamma} y^{s}\left(g_{\alpha}^{-1} \gamma z\right) u^{*}(\gamma) v,
$$

where $\alpha=1, \ldots, \kappa, v \in V_{\alpha} \subset V, g_{\alpha} \in \operatorname{PSL}(2, \mathbf{R})$, and $z \in \mathscr{H}$. These Eisenstein series are spanning up the continuous spectrum of the Laplacian on the Riemann surface. A Fourier expansion of the Eisenstein series (2.18) then yields

$$
e\left(z, s, \alpha, v_{l}(\alpha)\right)=P_{\beta} e\left(z, s, \alpha, v_{l}(\alpha)\right)=\sum_{m=-\infty}^{\infty} a_{j}(y, s) e^{2 \pi i m x}
$$

with the coefficients

$$
\begin{aligned}
& a_{0}(y, s)=\delta_{\alpha \beta} v_{l}(\alpha) y^{s}+\sqrt{\pi} \frac{\Gamma\left(s-\frac{1}{2}\right)}{\Gamma(s)} y^{1-s} \eta_{0}(s) \\
& a_{m}(y, s)=\frac{2 \pi^{s}}{\Gamma(s)}|m|^{s-\frac{1}{2}} \sqrt{y} K_{s-\frac{1}{2}}(2 \pi|k| y) \eta_{m}(s),(m \neq 0)
\end{aligned}
$$

with (see [64] for proper definitions and more details)

$$
\eta_{m}(s)=\sum_{\gamma \in \Gamma_{\alpha} \backslash \Gamma / \Gamma_{\beta}} \frac{P_{\beta} U^{*}(\gamma) v_{l}(\alpha)}{\left|c\left(g_{\alpha}^{-1} \gamma g_{\beta}\right)\right|^{2 s}} \exp \left[2 \pi i m \frac{d\left(g_{\alpha}^{-1} \gamma g_{\beta}\right)}{c\left(g_{\alpha}^{-1} \gamma g_{\beta}\right)}\right],
$$

with $c>0, d \bmod c,\left(\begin{array}{ll}* & * \\ c & d\end{array}\right) \in g_{\beta}^{-1} \Gamma g_{\alpha}$. The $\left(\kappa_{0} \times \kappa_{0}\right)$-matrix

$$
\mathscr{S}_{\alpha l, \beta k}=\sqrt{\pi} \frac{\Gamma\left(s-\frac{1}{2}\right)}{\Gamma(s)} \eta_{0}(s)
$$

is called scattering matrix and has the properties

i) $e(z, s, \alpha, v, \Gamma, U)=\mathscr{S}(s) e(z, 1-s, \alpha, v, \Gamma, U)$,

ii) $\mathscr{S}(s) \mathscr{S}(1-s)=1$ which is evidently true for $\kappa=1$ and is explicitly given in the matrix notation by

$$
\sum_{\beta=1}^{\kappa} \sum_{k=1}^{k_{\beta}} \mathscr{S}_{\alpha l, \beta k}(s) \mathscr{S}_{\beta k, \gamma m}(1-s)=\delta_{\alpha \gamma} \delta_{l m} .
$$


Let us denote $\Delta(s)=\operatorname{det}\left(\mathscr{S}_{\alpha l, \beta k}\right)$. The function $\Delta(s)$ has the following properties [64]

i) For any $p \in \mathbf{R} \Delta\left(\frac{1}{2}+i p\right) \neq 0$.

ii) It satisfies the functional equations $\Delta(s) \Delta(1-s)=1, \Delta^{*}\left(s^{*}\right)=\Delta(s)$, and in particular $\left|\Delta\left(\frac{1}{2}+i p\right)\right|=1$.

iii) It is regular in the half-plane $\Re(s)>\frac{1}{2}$ except for a finite number of poles on the interval of the real axis $s \in\left(\frac{1}{2}, 1\right]$ denoted by $\sigma_{1}, \sigma_{2}, \ldots, \sigma_{\mathscr{M}}$, which give due to the functional relation zeros, symmetric with respect to $s=\frac{1}{2}$ in the interval $s \in\left[0, \frac{1}{2}\right)$.

iv) In the half-plane $\mathfrak{R}(s)<\frac{1}{2} \Delta(s)$ has poles at $\rho=\beta+i \gamma\left(\beta<\frac{1}{2}\right)$ and the logarithmic derivative of $\Delta\left(\frac{1}{2}+i p\right)$ can be represented as

$$
-\frac{\Delta^{\prime}\left(\frac{1}{2}+i p\right)}{\Delta\left(\frac{1}{2}+i p\right)}=\sum_{\rho} \frac{1-2 \rho}{\left(\beta-\frac{1}{2}\right)^{2}+(p-\gamma)^{2}}+\text { const. }+O\left(\frac{1}{r^{2}}\right),
$$

for $p \rightarrow \infty, p \in \mathbf{R}$, with the summation over all poles $\rho=\beta+i \gamma$ of $\Delta(s)$ in the half-plane $\mathfrak{R}(s)<\frac{1}{2}$. Note that $\rho^{*}=\beta-i \gamma$ are poles as well, and due to the functional relation we have at $s=1-\rho$ and $s=1-\rho^{*}$, respectively, zeros for $\Delta(s)$.

Now the following theorem holds

Theorem 2.1 [64]. The Selberg trace formula on Riemann surfaces for hyperbolic, elliptic and parabolic conjugacy classes has the form:

$$
\begin{aligned}
\sum_{n=0}^{\infty} h\left(\frac{1}{4}+p_{n}^{2}\right)= & \frac{A}{4 \pi} \operatorname{dim} V \int_{-\infty}^{\infty} p \tanh \pi p h\left(\frac{1}{4}+p^{2}\right) d p \\
& +\sum_{\{\gamma\}} \sum_{k=1}^{\infty} \frac{\operatorname{tr}_{V}\left[U^{k}(\gamma)\right] l_{\gamma}}{2 \sinh \frac{k l_{\gamma}}{2}} g\left(k l_{\gamma}\right) \\
& +\sum_{\{R\}} \sum_{k=1}^{v-1} \frac{\operatorname{tr}_{V}\left[U^{k}(R)\right]}{4 v \sin (k \pi / v)} \int_{-\infty}^{\infty} h\left(\frac{1}{4}+p^{2}\right) \frac{\cosh [\pi(1-2 k / v) p]}{\cosh \pi p} d p \\
& +\frac{1}{4 \pi} \int_{-\infty}^{\infty} \frac{\Delta^{\prime}\left(\frac{1}{2}+i p\right)}{\Delta\left(\frac{1}{2}+i p\right)} h\left(\frac{1}{4}+p^{2}\right) d p-\frac{\kappa_{0}}{2 \pi} \int_{-\infty}^{\infty} h\left(\frac{1}{4}+p^{2}\right) \Psi(1+i p) d p \\
+ & \frac{1}{4}\left[\kappa_{0}-\operatorname{tr}(\mathscr{S})\right] h\left(\frac{1}{4}\right)-\left[\kappa_{0} \ln 2+\sum_{\alpha=1}^{v} \sum_{l=1+k_{\alpha}}^{\operatorname{dim} V} \ln \mid 1-e^{2 \pi i \theta_{l \alpha} \mid}\right] g(0) .
\end{aligned}
$$

The test function $h$ must satisfy the following properties

i) $h(p)=h\left(\frac{1}{4}+p^{2}\right)$ is an even function in $p$,

ii) $h(p)$ is analytic in the strip $\mathfrak{I}(p)<\frac{1}{2}+\varepsilon$ for some $\varepsilon>0$,

iii) and $h(p)$ vanishes according to $h(p)=O\left[1 /\left(1+p^{2}\right)^{2+\varepsilon}\right]$ for some $\varepsilon>0$ for $p \rightarrow \pm \infty$. 
Turning to the Selberg super-trace formula, let us introduce the Selberg super operator $L$ by

$$
\left.\begin{array}{rl}
L \phi(Z) & =\int_{\mathscr{H}^{(111)}} d V(W) k_{m}(Z, W) \phi(W), \\
k_{m}(Z, W) & =J^{M}(Z, W)\{\Phi[R(Z, W)]-r(Z, W) \Psi[R(Z, W)]\}, \\
J^{m}(Z, W) & =\left(\frac{z-\bar{w}+i \theta \bar{v}}{\bar{z}-w+i \bar{\theta} v}\right)^{\frac{m}{2}} .
\end{array}\right\}
$$

$k_{m}(Z, W)$ is the integral kernel of an operator valued function of the Dirac-Laplace operator $\square_{m}$ (respectively $\hat{\square}_{m}$ ), and $\Phi$ and $\Psi$ are sufficiently decreasing functions at infinity. $L$ is acting on super-automorphic functions $f(Z)$ with the property $f(\gamma Z)=j_{\gamma}^{m}(Z) f(Z)$, where $j_{\gamma}^{m}(Z)=\left(D \theta^{\prime} / \overline{D \theta^{\prime}}\right)^{\frac{m}{2}}$. Note that also some more general automorphic transformation property can be chosen such that $f(\gamma Z)$ $=U(\gamma) j_{\gamma}^{m}(Z) f(Z)$ and $U(\gamma)$ is some unitary representation of $\gamma$. Note furthermore the property $J^{m}(\gamma Z, \gamma W)=j_{\gamma}^{m}(Z) J^{m}(Z, W) j_{\gamma}^{-m}(W)$.

Let $f$ be a super-automorphic function with $f(\gamma Z)=U(\gamma) j_{\gamma}^{m}(Z) f(Z)$ and $g=L f$. Let $\mathscr{F}^{(1 \mid 1)}$ a fundamental domain of $T \in \Gamma$ whose body equals $\mathscr{F}$ (and is constructed in the same sense as the generalization $\mathscr{H}^{(1 \mid 1)}$ of $\mathscr{H}$ ), and $\sum_{\{T\}}$ denotes summation over distinct conjugacy classes. Then

$$
\begin{aligned}
g(Z) & =\int_{\mathscr{H}^{(11)}} d V(W) k_{m}(Z, W) f(W)=\sum_{\{T\}} \int_{\mathscr{F}^{(111)}} d V(W) k_{m}(Z, W) f(W) \\
& =\int_{\mathscr{F}^{(11))}} d V(W) K(Z, W) f(W),
\end{aligned}
$$

where

$$
K(Z, W)=\sum_{\{T\}} U(T) k_{m}(Z, T W) j_{T}^{m}(W)
$$

is the super-automorphic kernel. Let $Z_{\Gamma}(\gamma)$ the centralizer of a $\gamma \in \Gamma$. For $\operatorname{str}(L)$ we obtain on the one hand

$$
\operatorname{str}(L)=\sum_{n}\left[h\left(p_{n}^{B}\right)-h\left(p_{n}^{F}\right)\right],
$$

where $s_{n}^{B, F}=\frac{1}{2}+i p_{n}^{B, F}$ are the even and odd eigenvalues, respectively, of $\hat{\square}_{m}$, say. On the other we have by the usual procedure [34]

$$
\begin{aligned}
\operatorname{str}(L) & =\int_{\mathscr{F}^{(111)}} d V(W) K(Z, Z) \\
& =\sum_{T} \int_{\mathscr{F}^{(111)}} k_{m}(Z, T Z) U(T) j_{T}^{m}(Z) d V(Z) \\
& =\sum_{\{T\}} \sum_{\gamma \in Z_{\Gamma}(T) \backslash \Gamma} \int_{\mathscr{F}^{(111)}} k_{n}\left(Z, \gamma^{-1} T \gamma Z\right) U(T) j_{\gamma^{-1} T \gamma}^{m}(Z) d V(Z)
\end{aligned}
$$




$$
\begin{aligned}
& =\sum_{\{T\}} \sum_{\gamma \in Z_{\Gamma}(T) \backslash \Gamma} \int_{\mathscr{F}^{(111)}(\gamma)} k_{m}(\gamma Z, T \gamma Z) U(T) j_{T}^{m}(\gamma Z) d V(Z) \\
& =\sum_{\{\gamma\}} \operatorname{str}[U(\gamma)] \int_{\mathscr{F}^{(111)}(\gamma)} k_{m}(Z, \gamma Z) j_{\gamma}^{m}(W) d V(Z) \\
& =\sum_{\{\gamma\}} \operatorname{str}[U(\gamma)] \chi_{\gamma}^{m} A(\gamma)
\end{aligned}
$$

where $\mathscr{F}^{(1 \mid 1)}(\gamma)$ denotes the fundamental region of the super Fuchsian group $Z_{\Gamma}(\gamma)$, the centralizer of $\gamma \in \Gamma$. The expansion in hyperbolic, elliptic and parabolic conjugacy classes, respectively, yields

$$
\begin{aligned}
\operatorname{str}(L)= & \frac{i^{m}}{2} A \operatorname{dim} V \Phi(0)+\sum_{\substack{\{\gamma\} \\
\operatorname{str}(\gamma)+\chi_{\gamma}>2}} \operatorname{str}[U(\gamma)] \chi_{\gamma}^{m} A(\gamma) \\
& +\sum_{\substack{\{R\} \\
\operatorname{str}(R)+\chi_{R}<2}} \operatorname{str}[U(R)] \chi_{R}^{m} A(R)+\sum_{\substack{\{S\} \\
\operatorname{str}(S)+\chi_{s}=2}} \operatorname{str}[U(S)] \chi_{S}^{m} A(S),
\end{aligned}
$$

where the first term corresponds to the identity transformation (zero-length term) with $A(\gamma)$ given by ( $\gamma$ hyperbolic, elliptic, or parabolic, respectively)

$$
A(\gamma)=\chi_{\gamma}^{-m} \int_{\mathscr{F}^{(11)}(\gamma)} k_{m}(Z, \gamma Z) j_{\gamma}^{m}(W) d V(Z) .
$$

In Refs. $[7,27]$ the first two terms corresponding to the identity transformation and hyperbolic conjugacy classes, respectively, were calculated, i.e. (and furthermore $U \equiv 1$ )

$$
\begin{aligned}
& \sum_{n=0}^{\infty}\left[h\left(\frac{1+m}{2}+i p_{n}^{B}\right)-h\left(\frac{1+m}{2}+i p_{n}^{F}\right)\right] \\
& =-\frac{A}{4 \pi} \int_{0}^{\infty} \frac{g(u)-g(-u)}{\sinh \frac{u}{2}} \cosh \left(\frac{m}{2} u\right) d u \\
& \quad+\sum_{\{\gamma\}} \sum_{k=1}^{\infty} \frac{l_{\gamma} \chi_{\gamma}^{m k}}{2 \sinh \frac{k l_{\gamma}}{2}}\left[g\left(k l_{\gamma}\right)+g\left(-k l_{\gamma}\right)-\chi_{\gamma}^{k}\left(g\left(k l_{\gamma}\right) e^{-k l_{\gamma} / 2}+g\left(-k l_{\gamma}\right) e^{\left.k l_{\gamma}\right)}\right] .\right.
\end{aligned}
$$

Here the test function $h$ is required to have the properties
i) $h\left(\frac{1+m}{2}+i p\right) \in C^{\infty}(\mathbf{R})$,
ii) $h\left(\frac{1+m}{2}+i p\right)$ need not to be an even function in $p$ 
iii) $h\left(\frac{1+m}{2}+i p\right) \propto 1 /\left(1+|p|^{2}\right)^{\frac{1}{2}+\varepsilon}, \varepsilon>0$,

iv) $h\left(\frac{1+m}{2}+i p\right)$ is holomorphic in the $\operatorname{strip} \mathfrak{I}(p) \leqq 1+\frac{m}{2}+\varepsilon, \varepsilon>0$, to guarantee absolute convergence in the summation over $\{\gamma\}$.

The Fourier transformation $g$ of $h$ is given by

$$
\begin{aligned}
g(u)= & \frac{1}{2 \pi} \int_{-\infty}^{\infty} h\left(\frac{1+m}{2}+i p\right) e^{-i u p} d p \\
= & \frac{1}{4} \int_{4 \sinh ^{2} \frac{u}{2}}^{\infty} \frac{d x}{(x+4)^{m / 2}} \\
& \times\left\{\frac{\Psi(x)+2\left(e^{u}-1\right) \Phi^{\prime}(x)}{\sqrt{x-4 \sinh ^{2} \frac{u}{2}}}\left[\alpha_{+}^{m}(x, u)+\alpha_{-}^{m}(x, u)\right]\right. \\
& \left.-i m e^{u / 2} \Phi(x) \frac{\alpha_{+}^{m}(x, u)-\alpha_{-}^{m}(x, u)}{x+4}\right\},
\end{aligned}
$$

where $\alpha_{ \pm}^{m}(x, u)=\left(\sqrt{x-4 \sinh ^{2} \frac{u}{2}}-2 i \cosh \frac{u}{2}\right)^{m / 2}$. Specific trace formulae in particular for the heat kernel were considered by Aoki [1], Matsumoto, Uehara and Yasui $[42,43]$ and Uehara and Yasui [60], as well as an explicit evaluation for the energy dependent resolvent kernel for the operator $\hat{\square}_{m}^{2}$, including wave-functions (Oshima [48]). From the form of $g(u)$ an explicit formula for $\Phi(x)$ can be derived [27] which has the form

$$
i^{m} \Phi(x)=\frac{1}{\pi \sqrt{x+4}} \int_{x}^{\infty} \frac{d y}{\sqrt{y+4}} \int_{-\infty}^{\infty} Q_{1}^{\prime}\left(y+t^{2}\right)\left(\frac{\sqrt{y+t^{2}+4}-t}{\sqrt{y+t^{2}+4}+t}\right)^{m / 2} d t,
$$

with $Q_{1}(w)=\operatorname{coth} \frac{u}{2}[g(u)-g(-u)], w=4 \sinh ^{2} \frac{u}{2}$.

The above Selberg super-trace formula (2.33) is valid for discrete hyperbolic conjugacy classes and in this case the non-euclidean area of the ("bosonic") fundamental domain is given by $A=4 \pi(g-1)$, with $g$ the genus of the Riemann surface. In the case that elliptic fixpoints and cusp are present, this non-euclidean area is changed into (e.g. [34, Vol. II])

$$
A=2 \pi\left[2(g-1)+\sum_{j=1}^{s+\kappa}\left(1+\frac{1}{v_{j}}\right)\right],
$$

where $s$ denotes the number of inequivalent elliptic fixpoints and $\kappa$ the number of inequivalent cusps. $v_{j}$ denotes the order of the generators of the elliptic and parabolic subgroups $\Gamma_{j} \subset \Gamma(1 \leqq j \leqq s)$, respectively, i.e. for the elliptic $R_{j} \subset \Gamma$ $(1 \leqq j \leqq s)$; this means that $R_{j}^{v_{j}}=1$ for $(1 \leqq j \leqq s, 1<v<\infty)$, and for the order of 
the parabolic generators $S_{j}(s+1 \leqq j \leqq \kappa)$ it is assumed that $v_{j}=\infty$ $(j=1, \ldots, \kappa)$. The constraint $(2.8)$ is also altered due to the presence of elliptic fixpoints and cusps according to $[34,64]$

$$
\left(\gamma_{0} \gamma_{1}^{-1} \ldots \gamma_{2 g-2} \gamma_{2 g-1}^{-1}\right)\left(\gamma_{0}^{-1} \gamma_{1} \ldots \gamma_{2 g-2}^{-1} \gamma_{2 g-1}\right) R_{1} \ldots R_{s} S_{1} \ldots S_{k}=1_{2 \mid 1} .
$$

Our task will be now to incorporate the elliptic and parabolic conjugacy classes into the Selberg super-trace formula along the lines of the classical Selberg trace formula. We propose similarly as for the hyperbolic $\gamma \in \Gamma$ [cf. Eq. (2.7)] elliptic and parabolic $\gamma \in \Gamma$, and appropriate super fundamental domains $\mathscr{F}^{(1 \mid 1)}$. In particular, I propose a decomposition of an appropriate $\gamma \in \Gamma$ as follows

$\begin{aligned}(\gamma \in \Gamma \text { conjugate to }) & \left(\begin{array}{ccc}1 & 0 & 0 \\ 0 & 1 & 0 \\ 0 & 0 & \chi_{\gamma}\end{array}\right) \cdot\left(\begin{array}{ccc}N_{\gamma}^{\frac{1}{2}} & 0 & 0 \\ 0 & N_{\gamma}^{-\frac{1}{2}} & 0 \\ 0 & 0 & 1\end{array}\right) \\ & \times\left(\begin{array}{ccc}\cos \phi & -\sin \phi & 0 \\ \sin \phi & \cos \phi & 0 \\ 0 & 0 & 1\end{array}\right) \cdot\left(\begin{array}{lll}1 & n & 0 \\ 0 & 1 & 0 \\ 0 & 0 & 1\end{array}\right),\end{aligned}$

with $n \in \mathbf{N}$ and $0<\phi<\pi$. In the following an elliptic transformation (third factor) will be denoted by $R$, and a parabolic transformation (fourth factor) by $S$, respectively. The body $\mathscr{F}$ of such as fundamental domain $\mathscr{F}^{(1 \mid 1)}$ has according to [34] $4 g+2 s+2 \kappa$ sides, the boundaries being geodesics, of course. We also maintain the notion of $\chi_{\gamma}$ irrespective, whether $\gamma \in \Gamma$ is hyperbolic, elliptic or parabolic, respectively, and we choose $\chi_{\gamma}$ according to the spin structure of the super Riemann surface in question. For a super Riemann surface of genus $g$ there are obviously $2^{\text {(\# generators) }}=2^{(2 g+s+\kappa)}$ possible spin structures.

Only the $m=0$ case will be considered due to the quite involved inversion formulae for $m \neq 0$ for $\Psi(x)$.

For the incorporation of the elliptic and parabolic conjugacy classes, let us introduce some definitions, generalizing the notations of the classical to the "super"-case. Let $\kappa$ be the number of inequivalent cusps $Z_{j}(j=1, \ldots, \kappa), \Gamma_{j} \subset \Gamma$ the maximal subgroup which stabilizes it. Each subgroup $\Gamma_{j}$ is generated by a single parabolic element $S_{j}$. For each $j=1, \ldots, \kappa$ there exists a transformation $g_{j} \in \operatorname{OSp}(2, \mathbf{R}) /\{ \pm 1\}$ such that $g_{j} \infty=Z_{j}, \quad g_{j}^{-1} S_{j} g_{j} z=S_{\infty} Z=Z+1$ $\left(Z \in \mathscr{H}^{(1 \mid 1)}\right)$. Let $V$ be an $h$-dimensional vector space on $\Lambda_{\infty}$, i.e. $V=\Lambda_{\infty}^{h}$, and $U$ is a representation of $\Gamma$ which acts in $V$. For each $j=1, \ldots, \kappa$ we have a subspace $V_{j} \subset V$ of the operator $U\left(S_{j}\right)$, i.e. $V_{j}=\left\{v \in V \mid U\left(S_{j}\right) v=v\right\}$. Let $k_{j}=\operatorname{dim} V_{j}$ and $\kappa_{0}=\sum_{j=1}^{\kappa} k_{j}$. For each $\alpha(\alpha=1, \ldots, \kappa)$ one chooses a basis $v_{1}(\alpha), \ldots, v_{h}(\alpha)$ for $V$ such that

$$
U\left(S_{\alpha}\right)\left(1_{V}-P_{\alpha}\right) v_{l}(\alpha)=v_{l \alpha} v_{l}(\alpha),
$$

with $P_{\alpha}$ the projector on the subspace $V_{\alpha}$, and where it is supposed that we have the alternatives

$$
v_{l \alpha} v_{l}(\alpha)=\left\{\begin{array}{l}
0 \\
e^{2 \pi i \theta_{l \alpha}},
\end{array}\right.
$$

with the numbers $v_{l \alpha}, \theta_{l \alpha} \in \Lambda_{\infty}$, and $0<\left[\theta_{l \alpha}\right]_{\text {Body }}<1$. 
We consider super-automorphic functions $f(Z)$ with the property $f(\gamma Z)=U(\gamma) j_{\gamma}^{m}(Z) f(Z)$ for $\gamma \in \Gamma, \Gamma$ a super Fuchsian group. For $\kappa_{0} \geqq 1$ the contributions from the parabolic conjugacy classes must be regularized, i.e. the continuous spectrum of the operator $\square$ must be subtracted from the superautomorphic kernel. That is, the expression

$$
\operatorname{str} h(\square)=\int_{\mathscr{F}^{(111)}} K(Z, Z) d V(Z)
$$

does not exist in general and must be regularized.

\section{Elliptic Conjugacy Classes}

In order to discuss the incorporation of elliptic conjugacy classes elements into the Selberg super-trace formula we take the form of an elliptic element as proposed in Eq. (2.38). Therefore the effect of an elliptic transformation on super coordinates $Z=(z, \theta)$ is as follows:

$$
w=z^{\prime}=\frac{z \cos \phi-\sin \phi}{z \sin \phi+\cos \phi}, \quad v=\theta^{\prime}=\frac{\chi_{R} \theta}{z \sin \phi+\cos \phi} .
$$

This yields for the even and odd point-pair invariants, respectively

$$
\left.\begin{array}{rl}
R(Z, W) & =\sin ^{2} \phi\left[\frac{\left(1+x^{2}\right)^{2}}{y^{2}}+2\left(1+x^{2}\right)+y^{2}-4\right]\left(1-\frac{\theta \bar{\theta}}{y}\right) \\
& \equiv R_{0}\left(1-\frac{\theta \bar{\theta}}{y}\right), \\
r(Z, W) & =\frac{\theta \bar{\theta}}{y}\left(1-\chi_{R} \cos \phi\right) .
\end{array}\right\}
$$

Restricting to hyperbolic and elliptic conjugacy classes gives for the Selberg super-trace formula

$$
\begin{aligned}
\operatorname{str}(L)= & \sum_{\{\gamma\}} A(\gamma)=\frac{A}{2} \operatorname{dim} V \Phi(0) \\
& +\sum_{\substack{\{\gamma\} \\
\operatorname{sit}(\gamma)+\alpha_{\gamma}>2}} \operatorname{str}_{V}[U(\gamma)] A(\gamma)+\sum_{\substack{\{\boldsymbol{R}\} \\
\operatorname{str}(R)+\alpha_{R}<2}} \operatorname{str}_{V}[U(R)] A(R),
\end{aligned}
$$

with the first and second term as in Sect. II. Since $\int_{\mathscr{F}^{(11)}}=\int_{\mathscr{H}^{(111)}} / v[34]$ we obtain for $A(R)$ in the third term of Eq. (3.3),

$$
\begin{aligned}
A(R) & =\frac{1}{v} \int_{\mathscr{H}} \frac{d x d y d \theta d \bar{\theta}}{Y}[\Phi(R(Z, R Z))-r(Z, R Z) \Psi(R(Z, R Z))] \\
& =\frac{2}{v} \int_{0}^{\infty} \int_{0}^{\infty} \frac{d x d y}{y^{2}}\left[R_{0} \Phi^{\prime}\left(R_{0}\right)+\frac{1}{2} \Phi\left(R_{0}\right)+\left(1-\chi_{R} \cos \phi\right) \Psi\left(R_{0}\right)\right] .
\end{aligned}
$$


Following [34] we perform a variable substitution according to $t=\sin ^{2} \phi\left[\frac{\left(1+x^{2}\right)^{2}}{y^{2}}+2\left(1+x^{2}\right)+y^{2}-4\right], \quad d t=\frac{4 \sin \phi}{y} \sqrt{t+4 \sin ^{2} \phi} x d x$.

Applying Fubini's theorem, the domain of integration is mapped as $(0, \infty) \times(0, \infty) \rightarrow(0, \infty) \times\left(y_{1}, y_{2}\right)$, where $y_{1}$ and $y_{2}$ are given by

$$
y_{1,2}=\frac{\mp \sqrt{t}+\sqrt{t+4 s \sin ^{2} \phi}}{2 \sin \phi} \text {. }
$$

Note $y_{1} \cdot y_{2}=1$ and $x^{2}=\left(y-y_{1}\right)\left(y_{2}-y\right)$. Therefore we obtain

$$
\begin{aligned}
A(R)= & \frac{2}{v} \int_{0}^{\infty} \int_{0}^{\infty} \frac{d x d y}{y^{2}}\left[\frac{1}{2} \Phi(t)+t \Phi^{\prime}(t)+\left(1-\chi_{R} \cos \phi\right) \Psi(t)\right] \\
= & \frac{1}{2 v \sin \phi} \int_{0}^{\infty} \frac{d t}{\sqrt{t+4 \sin ^{2} \phi}}\left[\frac{1}{2} \Phi(t)+t \Phi^{\prime}(t)+\left(1-\chi_{R} \cos \phi\right) \Psi(t)\right] \\
& \times \int_{y_{1}}^{y_{2}} \frac{d y}{y \sqrt{y-y_{1}} \sqrt{y_{2}-y}} .
\end{aligned}
$$

By means of [21, p. 287]

$$
\begin{aligned}
& \int_{a}^{b}(x-a)^{\mu-1}(b-x)^{\nu-1}(x-c)^{-v-\mu} d x \\
& \quad=(b-a)^{\mu+v-1}(b-c)^{-\mu}(a-c)^{-v} B(\mu, v),
\end{aligned}
$$

where $B(x, y)$ is the Beta-function, we obtain for the $y$-integral the value $\pi$. By a further partial integration we obtain

$$
A(R)=\frac{\pi}{2 v \sin \phi} \int_{0}^{\infty} \frac{d t}{\sqrt{t+4 \sin ^{2} \phi}}\left[\left(1-\chi_{R} \cos \phi\right) \Psi(t)-\frac{2 \sin ^{2} \phi}{t+4 \sin ^{2} \phi} \Phi(t)\right] .
$$

From Sect. II we know the inversion formula for the function $\Phi$. It yields for $m=0$,

$$
\Phi(t)=-\frac{1}{\pi} \int_{0}^{\infty} \frac{Q_{1}(w)}{(w+4) \sqrt{w-t}} d w,
$$

and $Q_{1}$ as indicated. For an inversion for $\Psi$ we consider the quantity $Q_{2}(w)$ defined by

$$
Q_{2}(w)=\frac{g(u) e^{-u / 2}+g(-u) e^{u / 2}}{\cosh \frac{u}{2}}
$$

$\left(w=4 \sinh ^{2} \frac{u}{2}\right)$. We obtain therefore an inversion formula for $\Psi(t)$

$$
\Psi(t)=-\frac{1}{\pi} \int_{t}^{\infty} \frac{Q_{2}^{\prime}(w)}{\sqrt{w-t}} d w .
$$


Let us split the contributions for $A(R)$ in Eq. (3.9) into two parts, $A_{1}$ and $A_{2}$, according to the terms with $\Psi(t)$ and $\Phi(t)$, respectively. Using an elementary integration we obtain for $A_{1}(R)$,

$$
\begin{aligned}
A_{1}(R) & =\pi \frac{1-\chi_{R} \cos \phi}{2 v \sin \phi} \int_{0}^{\infty} \frac{\Psi(t)}{\sqrt{t+4 \sin ^{2} \phi}} d t \\
& =-\frac{1-\chi_{R} \cos \phi}{2 v \sin \phi} \int_{0}^{\infty} d w Q_{2}^{\prime}(w) \int_{0}^{w} \frac{d t}{\sqrt{-t^{2}+t\left(w-4 \sin ^{2} \phi\right)+4 w \sin ^{2} \phi}} \\
& =-\frac{1-\chi_{R} \cos \phi}{2 v \sin \phi} \int_{0}^{\infty} d w Q_{2}^{\prime}(w)\left(\frac{\pi}{2}+\arcsin \frac{w-4 \sin ^{2} \phi}{w+4 \sin ^{2} \phi}\right) \\
& =\frac{1-\chi_{R} \cos \phi}{v} \int_{0}^{\infty} \frac{d w}{\sqrt{w}} \frac{Q_{2}(w)}{w+4 \sin ^{2} \phi} \\
& =i \frac{1-\chi_{R} \cos \phi}{2 v \sin \phi \cos \phi} \int_{-\infty}^{\infty} \frac{\sinh \left[\left(p-\frac{i}{2}\right)(\pi-2 \phi)\right]}{\cosh \pi p},\left(\frac{1}{2}+i p\right) d p
\end{aligned}
$$

and in the last step I have used [21, p. 505]

$$
\int_{0}^{\infty} \frac{\cos (a x)}{\cosh \beta x+\cos \gamma x} d x=\frac{\pi}{\beta} \frac{\sinh \frac{a \gamma}{\beta}}{\sin \gamma \sinh \frac{a \pi}{\beta}} .
$$

Similarly by means of $[21$, p. 285$]$

$$
\int_{0}^{u}(x+\beta)^{v}(u-x)^{\mu-1} d x=\frac{\beta^{v} u^{\mu}}{\mu}{ }_{2} F_{1}\left(1,-v ; 1+\mu ;-\frac{u}{\beta}\right),
$$

and the property $\left[39\right.$, p. 38] ${ }_{2} F_{1}(-a, b ; b ;-z)=(1+z)^{a}$ I obtain

$$
\begin{aligned}
A_{2}(R) & =\frac{-\pi \sin \phi}{v} \int_{0}^{\infty} \frac{\Phi(t)}{\left(t+4 \sin ^{2} \phi\right)^{\frac{3}{2}}} d t \\
& =\frac{\sin \phi}{v} \int_{0}^{\infty} d w \frac{Q_{1}(w)}{w+4} \int_{0}^{w} \frac{d t}{\left(t+4 \sin ^{2} \phi\right)^{\frac{3}{2}} \sqrt{w-t}} \\
& =\frac{1}{v} \int_{0}^{\infty} \frac{\sqrt{w} Q_{1}(w)}{(w+4)\left(w+4 \sin ^{2} \phi\right)} d w \\
& =-\frac{i}{2 v \cos \phi} \int_{-\infty}^{\infty} \frac{\sinh [(\pi-2 \phi) p]}{\cosh \pi p} h\left(\frac{1}{2}+i p\right) d p,
\end{aligned}
$$

and in the last step use has been made of $[19$, p. 89]

$$
\int_{0}^{\infty} \frac{\sinh \frac{x}{2} \sin x y}{\cosh x+\cos a} d x=\frac{\pi}{2} \frac{\sin a y}{\sinh \frac{a}{2} \cosh \pi y} .
$$


Therefore we obtain for the elliptic contribution to the Selberg super-trace formula

$$
\begin{aligned}
& \sum_{\substack{\{R\} \\
\operatorname{str}(R)+\chi_{R}<2}} \operatorname{str}_{V}[U(R)] A(R)=\frac{i}{2} \sum_{\{R\}} \frac{\operatorname{str}_{V}[U(R)]}{v \cos (\pi / v)}\left\{\int_{-\infty}^{\infty} h\left(\frac{1}{2}+i p\right) d p\right. \\
& \left.\times\left[\frac{1-\chi_{R} \cos (\pi / v)}{\sin (\pi / v)} \frac{\sinh \left[\left(p-\frac{i}{2}\right)(\pi-2 \pi / v)\right]}{\cosh \pi p}-\frac{\sinh [(\pi-2 \pi / v) p]}{\cosh \pi p}\right]\right\} \\
= & \sum_{\{R\}} \sum_{k=1}^{v-1} \frac{\operatorname{str}_{V}\left[U^{k}(R)\right]}{v}\left\{\left(1-\chi_{R}^{k} \cos \frac{k \pi}{v}\right)\right. \\
& \left.\times \int_{0}^{\infty} \frac{g(u) e^{-u / 2}+g(-u) e^{u / 2}}{\cosh u-\cos (2 k \pi v)} d u+\int_{0}^{\infty} \frac{g(u)-g(-u)}{\cosh u-\cos (2 k \pi / v)} \sinh \frac{u}{2} d u\right\} .
\end{aligned}
$$

Note that we have $\lim _{\phi \rightarrow \frac{\pi}{2}} \frac{\sinh [\alpha(\pi-2 \phi)]}{\cos \phi}=2 \alpha$, and Eq. (3.18) is well-defined for $\phi \rightarrow \frac{\pi}{2}$ as well.

\section{Parabolic Conjugacy Classes}

Let us turn to the incorporation of parabolic elements. In the discussion of the parabolic conjugacy classes I first consider the case $\kappa=1$, for $v_{l \alpha} \neq 0$, in a onedimensional representation and $v_{l \alpha}=0$, respectively. The generalization to the general case then will be straightforward.

Let us therefore first assume that there is only one cusp. We have for the super-trace formula by including all conjugacy classes

$$
\begin{aligned}
\operatorname{str}(L)= & \frac{A}{2} \operatorname{dim} V \Phi(0)+\sum_{\substack{\{\gamma\} \\
\operatorname{str}(\gamma)+\chi_{\gamma}>2}} \operatorname{str}_{V}[U(\gamma)] A(\gamma) \\
& +\sum_{\substack{\{R\} \\
\operatorname{str}(R)+\chi_{R}<2}} \operatorname{str}_{V}[U(R)] A(R)+\sum_{\substack{\{S\} \\
\operatorname{str}(S)+\chi_{s}=2}} \operatorname{str}_{V}[U(S)] A(S),
\end{aligned}
$$

and we must investigate the fourth term. Again taking the proposition of Eq. (2.38) for parabolic elements we get for $S$ acting on super coordinates $Z=(z, \theta)$

$$
w=z^{\prime}=z+n, \quad v=\theta^{\prime}=\chi_{s} \theta .
$$

For the even and odd point-pair invariants $R(Z, W)$ and $r(Z, W)$, respectively, this yields

$$
R(Z, W)=\frac{n^{2}}{y^{2}}\left(1-\frac{\theta \bar{\theta}}{y}\right), \quad r(Z, W)=\frac{\theta \bar{\theta}}{y}\left(1-\chi_{s}\right)
$$

In order to be on the safe side we choose the fundamental domain for a parabolic transformation in such a way, that we consider the domain $[0,1] \times(0, \infty)$ for the $x$ and $y$-integrations, respectively, truncate it to $[0,1] \times\left(0, y_{M}\right)$, and consider finally the limit $y_{M} \rightarrow \infty$. Therefore with $A(S)=\lim _{y_{M} \rightarrow \infty} A_{y M}(S)$, 


$$
\begin{aligned}
A_{y_{M}}(S) & =\int_{0}^{1} d x \int_{0}^{y_{M}} d y \int \frac{d \theta d \bar{\theta}}{Y} \sum_{n \neq 0} k\left(Z, S^{n} Z\right) \\
& =\sum_{n \neq 0} \frac{1}{n} \int_{n / y_{M}}^{\infty} d u\left[\frac{1}{2} \Phi\left(u^{2}\right)+u^{2} \Phi^{\prime}\left(u^{2}\right)+\left(1-\chi_{S}\right) \Psi\left(u^{2}\right)\right] .
\end{aligned}
$$

We see clearly that this expression is divergent for $y_{M} \rightarrow \infty$. However, if $\kappa_{0}=0$, actually we have to consider by including $\operatorname{str}[U(S)]$

$$
A_{y_{M}}(S) \sum_{n \neq 0}^{\infty} \frac{e^{2 \pi i n \theta}}{n} \int_{n / y_{M}}^{\infty} d u\left[\frac{1}{2} \Phi\left(u^{2}\right)+u^{2} \Phi^{\prime}\left(u^{2}\right)+\left(1-\chi_{S}\right) \Psi\left(u^{2}\right)\right]
$$

(in the notation of Eq. (2.17) for one cusp) and the summation is convergent. Generally, for $\kappa_{0} \geqq 1$, we must apply the procedure of regularization as noted in Sect. II. The observed feature is due to the fact that generally operators with a continuous spectrum are not trace class. We must therefore consider a quantity like $\operatorname{str}\left(\right.$ kernel) $-\operatorname{str}\left(\right.$ continuous spectrum). We regularize $\operatorname{str}(h \square)$ for each $S_{j}$ according to

$$
\left.\begin{array}{l}
\operatorname{str} h(\square)=\int_{\mathscr{F}^{(1 \mid 1)}}[K(Z, Z)-H(Z, Z)] d V(Z) \\
H(Z, W)=-\frac{2}{\pi} \int_{-\infty}^{\infty} h\left(i p+\frac{1}{2}\right) E^{*}\left(i p+\frac{1}{2}, W\right) E\left(i p+\frac{1}{2}, Z\right) d p,
\end{array}\right\}
$$

$\left(\chi_{s}=1\right)$ and $E(s, Z)$ will be defined as a super Eisenstein series in complete analogy with the classical case, i.e. the continuous spectrum will be represented by an appropriate Eisenstein series along the lines of $[34,64]$. Span $\left[E\left(i p+\frac{1}{2}, Z\right)\right]$ will make up the continuous spectrum contribution of the Hilbert space $L^{2}\left(\Gamma \backslash \mathscr{H}^{(1 \mid 1)}\right)$, i.e. of the Hilbert space on the noncompact super Riemann surface.

Rearrangement of integration and summation yields in Eq. (4.4) [34, p. 204; 32, Theorem 422] ( $C=0.57721566490 \ldots$. Euler's constant $)$

$$
\begin{aligned}
A_{y_{M}}(S)= & 2 \sum_{n=1}^{\infty} \frac{1}{n} \int_{n / y_{M}}^{\infty}\left[\frac{1}{2} \Phi\left(u^{2}\right)+u^{2} \Phi^{\prime}\left(u^{2}\right)+\left(1-\chi_{S}\right) \Psi\left(u^{2}\right)\right] d u \\
= & 2 \int_{0}^{\infty} d u\left[\frac{1}{2} \Phi\left(u^{2}\right)+u^{2} \Phi^{\prime}\left(u^{2}\right)+\left(1-\chi_{S}\right) \Psi\left(u^{2}\right)\right] \sum_{1 \leqq n \leqq y_{M}} \frac{1}{n} \\
= & 2 \int_{0}^{\infty} d u\left[\frac{1}{2} \Phi\left(u^{2}\right)+u^{2} \Phi^{\prime}\left(u^{2}\right)+\left(1-\chi_{S}\right) \Psi\left(u^{2}\right)\right] \\
& \times\left[\ln u+\ln y_{M}+C+O\left(\frac{1}{\sqrt{u y_{M}}}\right)\right] \\
= & 2\left(1-\chi_{S}\right)\left\{\left(\ln y_{M}+C\right) \int_{0}^{\infty} \Psi\left(u^{2}\right) d u+\int_{0}^{\infty} \ln u \Psi\left(u^{2}\right) d u\right\} \\
& +2\left(\ln y_{M}+C\right) \int_{0}^{\infty}\left[\frac{1}{2} \Phi\left(u^{2}\right)+u^{2} \Phi^{\prime}\left(u^{2}\right)\right] d u \\
& +2 \int_{0}^{\infty} \ln u\left[\frac{1}{2} \Phi\left(u^{2}\right)+u^{2} \Phi^{\prime}\left(u^{2}\right)\right] d u+O\left(\frac{1}{\sqrt{y_{M}}}\right) .
\end{aligned}
$$


We consider the various terms. Firstly we have $\int_{0}^{\infty} \Psi\left(u^{2}\right) d u=g(0)$ and use has been made of $[21$, p. 287]

$$
\int_{a}^{b}(x-a)^{\mu-1}(b-x)^{v-1} d x=(b-a)^{\mu+v-1} B(\mu, v) .
$$

By a partial integration and using the integral [21, p. 538]

$$
\int_{0}^{1} x^{\mu-1}\left(1-x^{r}\right)^{v-1} \ln x d x=\frac{1}{r^{2}} B\left(\frac{\mu}{r}, v\right)\left[\Psi\left(\frac{\mu}{r}\right)-\Psi\left(\frac{\mu}{r}+v\right)\right],
$$

and some properties of the $\Psi(z)=\Gamma^{\prime}(z) / \Gamma(z)$ function (i.e. [21, p. 945] $\left.\Psi\left(\frac{1}{2}\right)=-C-2 \ln 2, \Psi(1)=-C\right)$ we obtain by collecting the relevant terms

$$
\begin{aligned}
A_{y_{M}}(S)= & 2\left(1-\chi_{S}\right)\left\{\left(\ln y_{M}+C-\ln 2\right) g(0)-\frac{1}{4} \int_{0}^{\infty} \ln w d Q_{2}(w)\right\} \\
& +\frac{1}{2} \int_{0}^{\infty}[g(u)-g(-u)] d u+O\left(\frac{1}{\sqrt{y_{M}}}\right) .
\end{aligned}
$$

Note that for $\kappa_{0}=0$ we obtain [34, p. 105],

$$
A_{\infty}(S)=-2\left(1-\chi_{S}\right) \ln \left|1-e^{2 \pi i \theta}\right| g(0)
$$

and no regularization is needed. Note furthermore the crucial dependence on $\chi_{s}$. If $\chi_{S}=1, A(S)=0$ for $\kappa_{0}=0$ and

$$
A(S) \equiv A_{\infty}(S)=\frac{1}{2} \int_{0}^{\infty}[g(u)-g(-u)] d u
$$

for $\chi_{S}=1, \kappa_{0} \neq 0$, and again no regularization is needed. Now consider

$$
\begin{aligned}
\frac{1}{4} \int_{0}^{\infty} \ln w d Q_{2}(w)= & -\frac{1}{4} \int_{0}^{\infty} \frac{g(u) e^{-u / 2}+g(-u) e^{u / 2}}{\cosh \frac{u}{2}} d u \\
& +\frac{1}{2} \int_{0}^{\infty} \ln \left(1-e^{-u}\right)\left[\frac{d}{d u} \frac{g(u) e^{-u / 2}+g(-u) e^{u / 2}}{\cosh \frac{u}{2}}\right] d u
\end{aligned}
$$

Using [19, p. 30]

$$
\int_{0}^{\infty} \frac{\cos x y}{\cosh a x} d x=\frac{\pi}{2 a} \frac{1}{\cosh \frac{\pi y}{2 a}}
$$

we get in the first integral in Eq. (4.13)

$$
\int_{0}^{\infty} \frac{g(u) e^{-u / 2}+g(-u) e^{u / 2}}{\cosh \frac{u}{2}} d u=\int_{-\infty}^{\infty} \frac{h\left(i p+\frac{1}{2}\right)}{\sinh \pi p} d p
$$


By a partial integration we get

$$
\begin{gathered}
\int_{0}^{\infty} \ln \left(1-e^{-u}\right)\left[\frac{d}{d u} \frac{g(u) e^{-u / 2}+g(-u) e^{u / 2}}{\cosh \frac{u}{2}}\right] d u \\
=-\frac{1}{\pi} \int_{-\infty}^{\infty} d p p h\left(\frac{1}{2}+i p\right) \int_{0}^{\infty} \ln \left(1-e^{-u}\right) \sin u p d u \\
+\frac{1}{2} \int_{0}^{\infty}[g(u)-g(-u)]\left(1-\tanh \frac{u}{2}\right) d u
\end{gathered}
$$

Using [21, p. 507]

$$
\int_{0}^{\infty} \sin \operatorname{ax}(1-\tanh \beta x) d x=\frac{1}{a}-\frac{\pi}{2 \beta \sinh \frac{a \pi}{2 \beta}},
$$

we obtain for the second integral

$$
\frac{1}{2} \int_{0}^{\infty}[g(u)-g(-u)]\left(1-\tanh \frac{u}{2}\right) d u=-\frac{i}{2} \int_{-\infty}^{\infty} h\left(i p+\frac{1}{2}\right)\left(\frac{1}{\pi p}-\frac{1}{\sinh \pi p}\right) d p .
$$

It remains to calculate one further integral, i.e. the first term on the right-hand side of Eq. (4.16). Using (4.9) again we get

$$
\int_{0}^{\infty} \ln \left(1-e^{-u}\right) \sin u p d u=-\frac{1}{2 p}[2 C+\Psi(1-i p)+\Psi(1+i p)] .
$$

Therefore combining all relevant terms we get

$$
\begin{aligned}
A_{y_{M}}(S)= & \left(1-\chi_{S}\right) \llbracket 2\left(\ln y_{M}+C-\ln 2\right) g(0)+\int_{0}^{\infty} g(-u) d u \\
& \left.-\int_{0}^{\infty} \ln \left(1-e^{-u}\right)\left\{\frac{d}{d u}[g(u)+g(-u)]\right\} d u\right] \\
& +\frac{1}{2} \int_{0}^{\infty}[g(u)-g(-u)] d u+O\left(\frac{1}{\sqrt{y_{M}}}\right) .
\end{aligned}
$$

The discussion in Sect. II suggests that we propose for each parabolic conjugacy class $(j=1, \ldots, \kappa)$ the following continuum regularization for the Selberg supertrace formula in the presence of cusps and $\kappa_{0} \geqq 1$,

$$
\begin{aligned}
\left.\operatorname{str}(L)\right|_{\text {cusp }_{j}} & =\int_{\mathscr{D}^{(11)}} K(Z, S Z) d V(Z) \\
& -c_{S_{j}} \frac{1-\chi_{S_{j}}}{\pi} \int_{-\infty}^{\infty} d p h\left(i p+\frac{1}{2}\right) \int_{\mathscr{H}^{(1 \mid 1)}} d V(Z) E_{j}\left(Z, i p+\frac{1}{2}\right) E_{j}^{*}\left(Z, i p+\frac{1}{2}\right)
\end{aligned}
$$


with some normalization constants $c_{s_{j}}$. Let us define the super Eisenstein series for one cusp and $\kappa_{0}=1$,

$$
E(Z, s):=\sum_{s \in \Gamma_{0} \backslash \Gamma}[Y(S Z)]^{s},
$$

with $\Gamma_{0}$ in the stabilizer of $\Gamma$ and with elements of the form of $\gamma_{\text {para }}$ of Eq. (2.38). This definition is completely analogous as in e.g. [34] or Kubota [36], respectively. Note that $Y(\gamma Z)$ is understood as

$$
Y\left[\left(\begin{array}{lll}
a & b & 0 \\
c & d & 0 \\
0 & 0 & \chi_{s}
\end{array}\right) Z\right]=\frac{Y}{|c z+d|^{2}} .
$$

We proceed similarly as in Ref. [36] and analyse $E(Z, s)$ by a Fourier transformation, i.e.

$$
E(Z, s)=\sum_{m=-\infty}^{\infty} a_{m}(Z, s) e^{2 \pi i m x}
$$

This yields for the coefficients $a_{m}(Z, s)$

$$
\begin{aligned}
a_{m}(Z, s) & =\int_{0}^{1} E(Z, s) e^{-2 \pi i m x} d x=\int_{0}^{1} \sum_{S \in \Gamma_{0} \backslash \Gamma} Y(S Z)^{s} e^{-2 \pi i m x} d x \\
& =Y^{s}+\int_{-\infty}^{\infty} \sum_{s \in \Gamma_{0} \backslash \Gamma} Y(S Z)^{s} e^{-2 \pi i m x} d x, S=\left(\begin{array}{ccc}
a & b & 0 \\
c & d & 0 \\
0 & 0 & \chi_{s}
\end{array}\right) \in \Gamma_{0} \backslash \Gamma / \Gamma_{0}, c \neq 0 \\
& =Y^{s}+\sum_{c, d} \int_{-\infty}^{\infty} \frac{Y^{s}}{|c z+d|^{s}} e^{-2 \pi i m x} d x \\
& =Y^{s}+\sum_{c} \frac{1}{|c|^{2 s}}\left(\sum_{d} e^{2 \pi i m d / c}\right) \int_{-\infty}^{\infty} \frac{Y^{s}}{\left|x^{2}+y^{2}\right|^{s}} e^{-2 \pi i m x} d x \\
& =Y^{s}+Y^{s} y^{1-2 s} \sum_{c} \frac{1}{|c|^{2 s}}\left(\sum_{d} e^{2 \pi i m d / c}\right)_{-\infty}^{\infty} \frac{e^{-2 \pi i m y t}}{\left(1+t^{2}\right)^{s}} d t
\end{aligned}
$$

The decomposition $\Gamma_{0} \backslash \Gamma / \Gamma_{0}$ guarantees that there are no $\gamma \in \Gamma$ left, containing parabolic transformations [36]. Using the integrals [21, p. 295, p. 426]

$$
\left.\begin{array}{l}
\int_{0}^{\infty} x^{\mu-1}\left(1+x^{2}\right)^{v-1} d x=\frac{1}{2} B\left(\frac{\mu}{2}, 1-v-\frac{\mu}{2}\right), \\
\int_{0}^{\infty}\left(\beta^{2}+x^{2}\right)^{v-\frac{1}{2}} \cos a x d x=\frac{\sqrt{\pi}}{\Gamma\left(\frac{1}{2}-v\right)}\left(\frac{2 \beta}{a}\right)^{v} K_{-v}(a \beta),
\end{array}\right\}
$$

we find for the $m=0$ and $m \neq 0$ term in $a_{m}(Z, s)$, respectively

$$
\left.\begin{array}{l}
\int_{-\infty}^{\infty} \frac{d t}{\left(1+t^{2}\right)^{s}}=\sqrt{\pi} \frac{\Gamma\left(s-\frac{1}{2}\right)}{\Gamma(s)}, \\
\int_{-\infty}^{\infty} \frac{e^{-2 \pi i m y t}}{\left(1+t^{2}\right) s} d t=\frac{2 \pi^{s}}{\Gamma(s)}(|m| y)^{s-\frac{1}{2}} K_{s-\frac{1}{2}}(2 \pi|m| y) .
\end{array}\right\}
$$


Therefore we obtain for $E(Z, s)$ the expansion

$$
\begin{aligned}
E(Z, s)= & \left(1+\frac{s}{2 y} \theta \bar{\theta}\right) \\
& \times\left[y^{s}+\phi_{0}(s) y^{1-s}+\sum_{m \neq 0} \phi_{m}(s) \sqrt{y} e^{2 \pi i m x} K_{s-\frac{1}{2}}(2 \pi|m| y)\right],
\end{aligned}
$$

and $\phi_{0}(s) \equiv \phi(s), \phi_{m}(s)$ respectively, are given by

$$
\left.\begin{array}{l}
\phi(s)=\sum_{c} \frac{1}{|c|^{2 s}}\left(\sum_{d} e^{2 \pi i m d / c}\right) \sqrt{\pi} \frac{\Gamma\left(s-\frac{1}{2}\right)}{\Gamma(s)} \\
\phi_{m}(s)=\sum_{c} \frac{1}{|c|^{2 s}}\left(\sum_{d} e^{2 \pi i m d / c}\right) \frac{2 \pi^{s}}{\Gamma(s)}|m|^{s-\frac{1}{2}}
\end{array}\right\}
$$

with $c>0, d \bmod c,\left(\begin{array}{ll}* & * \\ c & d\end{array}\right) \in S^{-1} \Gamma S$. Equation (4.28) shows nicely the general structure of even super functions which are eigenfunctions of the Laplace-Dirac operator $\square$ on the Poincaré super upper half-plane (see [7, 27,43] and Sect. II) and the super Eisenstein $E(z, s)$ series are therefore spanning up the continuous spectrum of the Dirac-Laplace operator $\square$ on the super Riemann surface, similarly as the Eisenstein series $e(z, s)$ are spanning up the continuous spectrum of the Laplacian $\Delta$ on the Riemann surface. We can therefore write

$$
E(Z, s)=\left(1+\frac{s}{2 y} \theta \bar{\theta}\right) e(z, s)
$$

with the Eisenstein series $e(z, s)$ given by

$$
e(z, s)=y^{s}+\phi_{0}(s) y^{1-s}+\sum_{m \neq 0} \phi_{m}(s) \sqrt{y} e^{2 \pi i m x} K_{s-\frac{1}{2}}(2 \pi|m| y)
$$

which is the classical Eisenstein series on the Poincare upper half-plane, see $[34,36]$ and Sect. II. This means that because $e(z, s)$ is an eigenfunction of the Laplacian $\Delta=-y^{2}\left(\partial_{x}^{2}+\partial_{y}^{2}\right)$ on the Poincare upper half-plane with Eigenvalue $\lambda=s(1-s), E(Z, s)$ is an eigenfunction of the Laplace-Dirac operator $\square$ on the Poincaré super upper half-plane with eigenvalue $\lambda=s$. Let us consider a domain $\mathscr{D}^{(1 \mid 1)}$ on the super upper half-plane whose body $\mathscr{D}=\left(\mathscr{D}^{(1 \mid 1)}\right)_{\text {Body }}$ is bounded. Then

$$
\begin{aligned}
& -2 \int_{\mathscr{D}^{(11)}} d V(Z)\left[E(Z, s) \square E^{*}(Z, t)-E^{*}(Z, t) \square E(Z, s)\right] \\
& \quad=-2\left(t^{*}-s\right) \int_{\mathscr{D}^{(112)}} d V(Z) E(Z, s) E^{*}(Z, t) \\
& =\left[s(1-s)-t^{*}\left(1-t^{*}\right)\right] \int_{\mathscr{D}} \frac{d x d y}{y^{2}} e(z, s) e^{*}(z, t) .
\end{aligned}
$$


Note that this special result of the function $E(Z, s)$ can be also achieved by considering two even super functions $U=u_{1}+\theta \bar{\theta} u_{2} / y$ and $V=v_{1}+\theta \bar{\theta} v_{2} / y$, respectively, and working out the identity

$$
\begin{aligned}
& \int_{\mathscr{D}_{(11)}} \frac{d z d \bar{z} d \theta d \bar{\theta}}{Y}\left[U(Z) \square V^{*}(Z)-V^{*}(Z) \square U(z)\right] \\
& \quad=-\int_{\mathscr{D}} d x d y\left[u_{1}(x, y)\left(\partial_{x}^{2}+\partial_{y}^{2}\right) v_{1}^{*}(x, y)-v_{1}^{*}(x, y)\left(\partial_{x}^{2}+\partial_{y}^{2}\right) u_{1}(x, y)\right] .
\end{aligned}
$$

Note also that odd super functions according to Refs. [7, 27] cancel out in this consideration. Equation (4.33) can then be tackled in the usual way by applying Green's theorem on it, etc. Setting $s=\frac{1}{2}+i p+\varepsilon$, Eq. (4.32) now gives $[34,36]$

$$
\int_{J_{y_{M}}} \frac{d x d y}{y^{2}}|e(z, s)|^{2}=\frac{y_{M}^{2 \varepsilon}-|\phi(s)|^{2} y_{M}^{-2 \varepsilon}}{2 \varepsilon}+2 \Re\left(\phi^{*}(s) \frac{y_{M}^{2 i p}}{2 i p}\right),
$$

where $J_{y_{M}}$ is a domain in $\mathscr{H}$ bounded by $y_{M}$ [34]. Therefore we can conclude that the following identity holds for $y_{M} \rightarrow \infty$ :

$$
\begin{aligned}
& -\frac{1}{\pi} \int_{-\infty}^{\infty} h\left(i p+\frac{1}{2}\right)\left[\int_{J_{J_{M}}^{(111)}} d V(Z)\left|E\left(Z, \frac{1}{2}+i p\right)\right|^{2}\right] d t \\
& =\frac{1}{2 \pi} \int_{-\infty}^{\infty} h\left(i p+\frac{1}{2}\right)\left[\int_{J_{y_{M}}} \frac{d x d y}{y^{2}}\left|e\left(z, \frac{1}{2}+i p\right)\right|^{2}\right] d t \\
& =2 g(0) \ln y_{M}-\frac{1}{2 \pi} \int_{-\infty}^{\infty} h\left(i p+\frac{1}{2}\right) \frac{\phi^{\prime}\left(\frac{1}{2}+i p\right)}{\phi\left(\frac{1}{2}+i p\right)} d p+\frac{1}{2} \phi\left(\frac{1}{2}\right) h\left(\frac{1}{2}\right)+O(1) .
\end{aligned}
$$

In Eq. (4.21) we choose $c_{s}=-1$ which gives the regularization

$$
\begin{aligned}
\left.\operatorname{str}(L)\right|_{\text {cusp }}= & \int_{\mathscr{D}^{(11)}} K(Z, S Z) d V(Z) \\
& +2 \frac{1-\chi_{S}}{\pi} \int_{\infty}^{\infty} d p h\left(i p+\frac{1}{2}\right) \int_{\mathscr{C}^{(11)}} d V(Z) E\left(Z, i p+\frac{1}{2}\right) E^{*}\left(Z, i p+\frac{1}{2}\right) .
\end{aligned}
$$

The first term of Eq. (4.35) and the corresponding term in Eq. (4.20) exactly cancel and the parabolic contribution to the Selberg super-trace formula are therefore given by $\left(\kappa_{0}=1\right)$

$$
\begin{aligned}
A(S)= & \left(1-\chi_{S}\right) \llbracket-2 g(0) \ln 2+\frac{1}{2 \pi} \int_{-\infty}^{\infty} h\left(i p+\frac{1}{2}\right) \frac{\phi^{\prime}\left(\frac{1}{2}+i p\right)}{\phi\left(\frac{1}{2}+i p\right)} d p-\frac{1}{2} \phi\left(\frac{1}{2}\right) h\left(\frac{1}{2}\right) \\
& +\int_{0}^{\infty} g(-u) d u-\int_{0}^{\infty} \ln \left(1-e^{-u}\right)\left\{\frac{d}{d u}[g(u)+g(-u)]\right\} d u \rrbracket \\
& +\frac{1}{2} \int_{0}^{\infty}[g(u)-g(-u)] d u .
\end{aligned}
$$

This completes the calculation of the Selberg super-trace formula in the case of the presence of parabolic elements in the corresponding Fuchsian super group (one cusp). 
Now consider the general case of the presence of several cusps and some numbers $\theta_{l a}$ as in Eq. (2.40). Of course, we must only regularize the superautomorphic kernel by the incorporation of $E(Z, s)$ whenever $\chi_{s_{j}}=-1$ $(j \in\{1, \ldots, \kappa\})$. We consider

$$
E(Z, s, \alpha, v)=\sum_{\gamma \in \Gamma_{\alpha} \backslash \Gamma} Y^{s}\left(g_{\alpha}^{-1} \gamma Z\right) U^{*}(\gamma) v(\alpha) .
$$

We assume a Fourier expansion according to

$$
E_{\beta}\left(g_{\beta} Z, s, \alpha, v_{l}(\alpha)\right)=P_{\beta} E\left(g_{\beta} Z, s, \alpha, v_{l}(\alpha)\right)=\sum_{m=-\infty}^{\infty} a_{m}(Y, s) e^{2 \pi i m x}
$$

with the coefficients $a_{m}(Y, s)$ (compare $[36,64]$ )

$$
\begin{aligned}
a_{m}(Y, s) & =\int_{0}^{1} \sum_{\gamma \in \Gamma_{\alpha} \backslash \Gamma} Y^{s}\left(g_{\alpha}^{-1} \gamma g_{\beta} Z\right) P_{\beta} U^{*}(\gamma) v_{l}(\alpha) e^{-2 \pi i m x} d x \\
& =\sum_{\gamma \in \Gamma_{\alpha} \backslash \Gamma / \Gamma_{\beta}} P_{\beta} U^{*}(\gamma) v_{l}(\alpha) \int_{-\infty}^{\infty} Y^{s}\left(g_{\alpha}^{-1} \gamma g_{\beta} Z\right) e^{-2 \pi i m x} d x \\
& =\left(1+\frac{s}{2 y} \theta \bar{\theta}\right) e_{\beta}\left(g_{\beta} z, s, \alpha, v_{l}(\alpha)\right),
\end{aligned}
$$

with $e_{\beta}\left(g_{\beta} Z, s, \alpha, v_{l}(\alpha)\right)$ as in Eq. (2.19) and all the results deduced from the properties of the classical Eisenstein series can be used appropriately in the Selberg super-trace formula.

First of all, repeating the calculations above, we have instead of Eq. (4.11) now

$$
A_{y_{M}}(S)=-2\left(1-\chi_{S}\right) \ln |\operatorname{sdet}(1-U(S))| g(0) .
$$

Next we have for $\kappa_{0} \geqq 1$ and $s=\frac{1}{2}+i p+\varepsilon$,

$$
\begin{aligned}
& \lim _{\varepsilon \rightarrow 0} \int_{J_{y_{M}}} \frac{d x d y}{y^{2}}\left|e_{\beta}\left(g_{\beta} z, s, \alpha, v_{l}(\alpha)\right)\right|^{2} \\
& =\lim _{\varepsilon \rightarrow 0} \frac{\kappa_{0} y_{M}^{2 \varepsilon}-\sum_{\alpha l} \sum_{\beta k}\left|\mathscr{S}_{\alpha l, \beta, k}\left(\frac{1}{2}+i p+\varepsilon\right)\right|^{2} y_{M}^{-2 \varepsilon}}{2 \varepsilon} \\
& =2 \kappa_{0} \ln y_{M}-\frac{\Delta^{\prime}\left(\frac{1}{2}+i p\right)}{\Delta\left(\frac{1}{2}+i p\right)}+2 \sum_{\alpha l} \mathfrak{R}\left[\mathscr{S}_{\alpha l, \alpha l}^{*}\left(\frac{1}{2}+i p\right) \frac{y_{M}^{2 i p}}{2 i p}\right] .
\end{aligned}
$$

Therefore consequently

$$
\begin{aligned}
-\frac{2}{\pi} & \int_{-\infty}^{\infty} h\left(i p+\frac{1}{2}\right) \int_{\mathscr{F}^{(11))}} d V(Z)\left|E\left(Z, \frac{1}{2}+i p\right)\right|^{2} \\
= & \frac{1}{2 \pi} \int_{-\infty}^{\infty} h\left(i p+\frac{1}{2}\right) \int_{\mathscr{F}} \frac{d x d y}{y^{2}}\left|e\left(z, \frac{1}{2}+i p\right)\right|^{2} \\
= & \frac{1}{2} h\left(\frac{1}{2}\right) \operatorname{tr}\left[\mathscr{S}\left(\frac{1}{2}\right)\right]+2 \kappa_{0} g(0) \ln y_{M} \\
& -\frac{1}{2 \pi} \int_{-\infty}^{\infty} h\left(i p+\frac{1}{2}\right) \frac{\Delta^{\prime}\left(\frac{1}{2}+i p\right)}{\Delta\left(\frac{1}{2}+i p\right)} d p+O(1) .
\end{aligned}
$$


Summarizing, we have shown

Theorem 4.1. The Selberg super-trace formula on super Riemann surfaces for hyperbolic, elliptic and parabolic conjugacy classes has the form:

$$
\begin{aligned}
\sum_{n=0}^{\infty}[ & \left.\left(\frac{1}{2}+i p_{n}^{B}\right)-h\left(\frac{1}{2}+i p_{n}^{F}\right)\right] \\
= & i \operatorname{dim} V \frac{A}{4 \pi} \int_{-\infty}^{\infty} h\left(i p+\frac{1}{2}\right) \tanh \pi p d p \\
& +\sum_{\{\gamma\}} \sum_{k=1}^{\infty} \frac{\operatorname{str}\left[U^{k}(\gamma)\right] l_{\gamma}}{2 \sinh \frac{k l_{\gamma}}{2}}\left[g\left(k l_{\gamma}\right)+g\left(-k l_{\gamma}\right)-\chi_{\gamma}^{k}\left(g\left(k l_{\gamma}\right) e^{-k l_{y} / 2}+g\left(-k l_{\gamma}\right) e^{k l_{\gamma} / 2}\right)\right] \\
& +\sum_{\{R\}} \sum_{k=1}^{v-1} \frac{\operatorname{str}\left[U^{k}(R)\right]}{v}\left\{\left(1-\chi_{R}^{k} \cos \frac{k \pi}{v}\right) \int_{0}^{\infty} \frac{g(u) e^{-u / 2}+g(-u) e^{u / 2}}{\cosh u-\cos (2 k \pi / v)} d u\right. \\
& -2\left[\int_{0}^{\infty} \frac{g(u)-g(-u)}{\cosh u-\cos (2 k \pi / v)} \sinh \frac{u}{2} d u\right\} \\
& -\frac{\kappa_{-}}{2} h\left(\frac{1}{2}\right) \ln \left[\mathscr{S}\left(\frac{1}{2}\right)\right]+\kappa_{-} \int_{0}^{\infty} g(-u) d u \\
& +\frac{\kappa_{-}}{2 \pi} \int_{-\infty}^{\infty} h\left(i p+\frac{1}{2}\right) \frac{\Delta^{\prime}\left(\frac{1}{2}+i p\right)}{\Delta\left(\frac{1}{2}+i p\right)} d p \\
& +\frac{\tilde{\kappa}_{0}}{2 \pi} \int_{-\infty}^{\infty} h\left(i p+\frac{1}{2}\right)[\Psi(1+i p)+\Psi(1-i p)] d p \\
& +\frac{\kappa_{0}}{2} \int_{0}^{\infty}[g(u)-g(-u)] d u,
\end{aligned}
$$

where $\sum_{\left\{S_{j}\right\}} \kappa_{j}\left(1-\chi_{S_{j}}\right), \kappa_{ \pm}=\sum_{\left\{S_{j}\right\}}\left(1 \pm \chi_{S_{j}}\right)$ and the other terms similarly interpreted. In particular the $p$-integral over $\Delta^{\prime}\left(\frac{1}{2}+i p\right) / \Delta\left(\frac{1}{2}+i p\right)$ is only present if $\tilde{\kappa}_{0} \neq 0$. Of course, $g(u)$ and $h\left(\frac{1}{2}+i p\right)$ can be replaced by each other through their corresponding Fourier transforms, cf. Eqs. $(2.34,3.18)$, respectively. This concludes the discussion.

\section{Selberg Super-Zeta Functions}

In order to discuss the Selberg super-zeta functions, let us first introduce the classical Selberg zeta function $Z(s)$ defined by

$$
Z(s)=\prod_{\gamma \in \Gamma} \prod_{k=0}^{\infty}\left[1-e^{-(s+k) l_{\nu}}\right], \quad \mathfrak{R}(s)>1 .
$$


As long as only the elliptic terms are maintained, the principal analytic structure [i.e. zeros of $Z(s)$ ] as e.g. in Refs. [34, Vol. 1, 38, 57] is not very much altered. However, the parabolic terms give rise to additional poles and do in fact matter a lot, see Ref. [64].

A functional equation can be derived which has the form $[34,57,64]$

$$
Z(1-s)=\Delta(s) \exp \left\{-A \operatorname{dim} V \int_{0}^{s-\frac{1}{2}} \mathrm{t} \tan \pi t d t\right\}\left[\frac{\Gamma\left(\frac{3}{2}-s\right)}{\Gamma\left(s+\frac{1}{2}\right)}\right]^{\kappa_{0}} \Psi_{Z}(s) Z(s),
$$

where $\Psi_{Z}(s)$ is defined as

$$
\begin{aligned}
\Psi_{Z}(s)= & \exp \left\{\sum_{\{R\}} \sum_{k=1}^{v-1} \frac{\operatorname{tr}_{V}\left[U^{k}(R)\right]}{v \sin (2 k \pi / v)} \sum_{l=1}^{\infty}\left(2 l \frac{k \pi}{v}\right) \ln \mid \frac{(s+l)(s+l-1)}{(s-l)(s-l-1)}\right] \\
& \left.\left.+(1-2 s)\left[\kappa_{0} \ln 2+\sum_{\alpha=1}^{v} \sum_{l=1+k_{\alpha}}^{\operatorname{dim} V} \ln \mid 1-e^{2 \pi i \theta_{l \mid l}}\right]\right]-i \arg \Delta\left(\frac{1}{2}\right)\right\} .
\end{aligned}
$$

Note the relation

$$
\begin{aligned}
R(s) R(-s): & =\frac{Z(s) Z(-s)}{Z(1+s) Z(1-s)} \\
& =(2 \sin \pi s)^{A \operatorname{dim} V / \pi}\left(\frac{1}{4}-s^{2}\right)^{-\kappa_{0}} \frac{\Delta(1+s)}{\Delta(s)} \frac{1}{\Psi(s) \Psi(-s)} .
\end{aligned}
$$

Let us consider the two Selberg super-zeta functions $Z_{0}$ and $Z_{1}$, respectively, defined by $[7,27]$

$$
\begin{array}{ll}
Z_{0}(s)=\prod_{\{\gamma\}} \prod_{k=0}^{\infty} \operatorname{sdet}\left[1_{V}-U(\gamma) e^{-(s+k) l_{\nu}}\right], & \mathfrak{R}(s)>1, \\
Z_{1}(s)=\prod_{\{\gamma\}} \prod_{k=0}^{\infty} \operatorname{sdet}\left[1_{V}-U(\gamma) \chi_{\gamma} e^{-(s+k) l_{\nu}}\right], & \Re(s)>1 .
\end{array}
$$

For convenience we will consider the functions

$$
R_{0}(s)=\frac{Z_{0}(s)}{Z_{0}(s+1)}, \quad R_{1}(s)=\frac{Z_{1}(s)}{Z_{1}(s+1)}, \quad \Re(s)>1,
$$

and the analytic properties of the $Z_{0,1}$-functions can be easily derived from the $R_{0,1}$-functions. As we shall see, only functional relations for the $R_{0,1}$-functions can be derived, but not for the $Z_{0,1}$-functions.

1. The Selberg super-zeta function $R_{1}$. We first discuss the function $Z_{1}(s)$. In order to do this we choose the test function [27] $(\Re(s, a)>1)$

$$
h_{1}\left(\frac{1}{2}+i p, s, a\right)=2 i p\left(\frac{1}{s^{2}+p^{2}}-\frac{1}{a^{2}+p^{2}}\right),
$$

with the Fourier transformed function $g_{1}(u)$ given by

$$
g_{1}(u, s, a)=\operatorname{sign}(u)\left(e^{-s|u|}-e^{-a|u|}\right) .
$$


A regularization term is needed to match the requirements of a valid test function for the trace formula. The hyperbolic- and zero-length term have already been evaluated in [27] with result

$i \operatorname{dim} V \frac{A}{4 \pi} \int_{-\infty}^{\infty} h_{1}\left(i p+\frac{1}{2}, s, a\right) \tanh \pi p d p=\operatorname{dim} V \frac{A}{\pi}\left[\Psi\left(s+\frac{1}{2}\right)-\Psi\left(a+\frac{1}{2}\right)\right]$,

$\sum_{\{\gamma\}} \sum_{k=1}^{\infty} \frac{l_{\gamma}}{2 \sinh \frac{k l_{\gamma}}{2}}\left[g_{1}\left(k l_{\gamma}, s, a\right)+g_{1}\left(-k l_{\gamma}, s, a\right)\right.$

$\left.-\chi_{\gamma}^{k}\left(g_{1}\left(k l_{\gamma}, s, a\right) e^{-k l_{\gamma} / 2}+g_{1}\left(-k l_{\gamma}, s, a\right) e^{k l_{\gamma}}\right)\right]=\frac{R_{1}^{\prime}(s)}{R_{1}(s)}-\frac{R_{1}^{\prime}(a)}{R_{1}(a)}$.

Next we consider the elliptic terms. By the use of [21, p. 357]

$$
\int_{0}^{\infty} \frac{e^{-\mu x}}{\cosh x-\cos t} d x=\frac{2}{\sin t} \sum_{l=1}^{\infty} \frac{\sin l t}{\mu+l}
$$

we obtain

$$
\begin{aligned}
& -\int_{0}^{\infty} \frac{g_{1}(u, s, a) e^{-u / 2}+g_{1}(-u, s, a) e^{u / 2}}{\cosh ^{2} \frac{u}{2}-\cos ^{2} \phi} d u \\
& =\frac{4}{\cos \phi} \sum_{l=0}^{\infty} \cos [(2 l+1) \phi]\left(\frac{1}{s+l+\frac{1}{2}}-\frac{1}{a+l+\frac{1}{2}}\right) .
\end{aligned}
$$

Let us turn to the parabolic terms. Quite easily we have $g_{1}(0)=0$, and $h_{1}\left(\frac{1}{2}, s, a\right)=0$. Furthermore

$$
\left.\begin{array}{c}
\int_{-\infty}^{\infty} h_{1}\left(i p+\frac{1}{2}, s, a\right)[\Psi(1+i p)+\Psi(1-i p)]=0, \\
\int_{0}^{\infty} g_{1}( \pm u, s, a) d u= \pm\left(\frac{1}{s}-\frac{1}{a}\right) .
\end{array}\right\}
$$

Let us consider the contour integral [64]

$$
\begin{aligned}
& \frac{1}{2 \pi i} \oint_{C} \frac{\Delta^{\prime}(z)}{\Delta(z)}\left[\frac{2\left(z-\frac{1}{2}\right)}{s^{2}-\left(z-\frac{1}{2}\right)^{2}}-\frac{2\left(z-\frac{1}{2}\right)}{a^{2}-\left(z-\frac{1}{2}\right)^{2}}\right] d z \\
& \quad=-\frac{1}{2 \pi i} \oint_{C} \frac{\Delta^{\prime}(z)}{\Delta(z)}\left[\frac{1}{z-\left(s+\frac{1}{2}\right)}+\frac{1}{z+\left(s-\frac{1}{2}\right)}-\frac{1}{z-\left(a+\frac{1}{2}\right)}+\frac{1}{z+\left(a-\frac{1}{2}\right)}\right],
\end{aligned}
$$

where the contour is running from $z=\frac{1}{2}-i R$ to $z=\frac{1}{2}+i R$ on the line $\mathfrak{R}(z)=\frac{1}{2}$, and closed by the semi-circle going through the points $z=\frac{1}{2}-i R, z=\frac{1}{2}-R$, $z=\frac{1}{2}+i R$ and the points are given in the direction they are tranversed by the contour. Considering $R \rightarrow \infty$, the integral over the semi-circle vanishes due to the properties of the logarithmic derivative of $\Delta(z)$ [cf. Eq. (2.24)] and the choice of the 
test function. The integral over the line then yields by the Cauchy residue theorem [64]

$$
\begin{aligned}
& \frac{1}{2 \pi} \int_{-\infty}^{\infty} h_{1}\left(i p+\frac{1}{2}, s, a\right) \frac{\Delta^{\prime}\left(\frac{1}{2}+i p\right)}{\Delta\left(\frac{1}{2}+i p\right)} d p=-\frac{\Delta^{\prime}\left(s+\frac{1}{2}\right)}{\Delta\left(s+\frac{1}{2}\right)}+\frac{\Delta^{\prime}\left(a+\frac{1}{2}\right)}{\Delta\left(a+\frac{1}{2}\right)} \\
& -\sum_{j=1}^{\mathscr{M}}\left[\frac{1}{s-\left(\sigma_{j}-\frac{1}{2}\right)}-\frac{1}{s+\left(\sigma_{j}-\frac{1}{2}\right)}-\frac{1}{a-\left(\sigma_{j}-\frac{1}{2}\right)}+\frac{1}{a+\left(\sigma_{j}-\frac{1}{2}\right)}\right] \\
& \quad-\sum_{\rho, \beta<\frac{1}{2}}\left[\frac{1}{s-\left(\rho-\frac{1}{2}\right)}-\frac{1}{s+\left(\rho-\frac{1}{2}\right)}-\frac{1}{a-\left(\rho-\frac{1}{2}\right)}+\frac{1}{a+\left(\rho-\frac{1}{2}\right)}\right] .
\end{aligned}
$$

Therefore we obtain the Selberg super-trace formula for the test function $h_{1}\left(i p+\frac{1}{2}, s, a\right)$ as follows:

$$
\begin{aligned}
& \frac{R_{1}^{\prime}(s)}{R_{1}(s)}-\frac{R_{1}^{\prime}(a)}{R_{1}(a)} \\
& =-\Delta n_{0}^{(0)}\left(\frac{1}{s^{2}-\frac{1}{4}}-\frac{1}{a^{2}-\frac{1}{4}}\right) \\
& \quad+2 \sum_{n=1}^{\infty}\left[\frac{\lambda_{n}^{B}-\frac{1}{2}}{s^{2}-\left(\lambda_{n}^{B}-\frac{1}{2}\right)^{2}}-\frac{\lambda_{n}^{B}-\frac{1}{2}}{a^{2}-\left(\lambda_{n}^{B}-\frac{1}{2}\right)^{2}}-\frac{\lambda_{n}^{F}-\frac{1}{2}}{s^{2}-\left(\lambda_{n}^{F}-\frac{1}{2}\right)^{2}}+\frac{\lambda_{n}^{F}-\frac{1}{2}}{a^{2}-\left(\lambda_{n}^{F}-\frac{1}{2}\right)^{2}}\right] \\
& \quad-2 \sum_{\{R\}}^{v-1} \frac{\operatorname{str}\left[U^{k}(R)\right]}{v} \chi_{R}^{k} \sum_{l=0}^{\infty} \cos \left[(2 l+1) \frac{k \pi}{v}\right]\left(\frac{1}{s+l+\frac{1}{2}}-\frac{1}{a+l+\frac{1}{2}}\right) \\
& \quad-\frac{A \operatorname{dim} V}{\pi}\left[\Psi\left(s+\frac{1}{2}\right)-\Psi\left(a+\frac{1}{2}\right)\right]+\kappa_{-}\left(\frac{1}{s}-\frac{1}{a}\right) \\
& \quad-\kappa_{0}\left(\frac{1}{s}-\frac{1}{a}\right)+\kappa_{-} \frac{\Delta^{\prime}\left(s+\frac{1}{2}\right)}{\Delta\left(s+\frac{1}{2}\right)}-\kappa_{-} \frac{\Delta^{\prime}\left(a+\frac{1}{2}\right)}{\Delta\left(a+\frac{1}{2}\right)} \\
& \quad+\kappa_{-} \sum_{j=1}^{\mathscr{M}}\left[\frac{1}{s-\left(\sigma_{j}-\frac{1}{2}\right)}-\frac{1}{s+\left(\sigma_{j}-\frac{1}{2}\right)}-\frac{1}{a-\left(\sigma_{j}-\frac{1}{2}\right)}+\frac{1}{a+\left(\sigma_{j}-\frac{1}{2}\right)}\right] \\
& \quad+\kappa_{-} \sum_{\rho, \beta<\frac{1}{2}}\left[\frac{1}{s-\left(\rho-\frac{1}{2}\right)}-\frac{1}{s+\left(\rho-\frac{1}{2}\right)}-\frac{1}{a-\left(\rho-\frac{1}{2}\right)}+\frac{1}{a+\left(\rho-\frac{1}{2}\right)}\right]
\end{aligned}
$$

Here $\Delta n_{0}^{(0)}=n_{0}^{B}-n_{0}^{F}$ denotes the difference between the number of even- and odd zero-modes of the Dirac-Laplace operator $\square$. According to Ref. [47] $\Delta n_{0}^{(0)}=1-2 q$ with $q=\operatorname{dim} \operatorname{ker} \bar{\partial}_{1}$ and $\bar{\partial}_{p}^{\dagger}=-y^{2} \partial_{z}+\frac{1}{2} p y$. From Eq. (5.17) we read off 
Theorem 5.1. The Selberg super-zeta function $R_{1}(s)$ is a meromorpic function on $\Lambda_{\infty}$ and has furthermore the following properties:

A) The Selberg super-zeta function $R_{1}(s)$ has "trivial" zeros at the following points and nowhere else:

i) $s=-\frac{1}{2}-l(l=0,1,2, \ldots)$ and the multiplicity of these zeros is given by

$$
\# N_{l}=\frac{A \operatorname{dim} V}{\pi}-2 \sum_{\{R\}} \sum_{k=1}^{v-1} \frac{\operatorname{str}\left[U^{k}(R)\right]}{v} \chi_{R}^{k} \sum_{l=0}^{\infty} \cos \left[(2 l+1) \frac{k \pi}{v}\right] \text {. }
$$

Note that for $l=0$ there is an additional $\Delta n_{0}^{(0)}$ term coming from the super-trace of $h_{1}\left(i p+\frac{1}{2}, s, a\right)$ for $\lambda=0$. Note also that if $\# N_{l}<0$, we have poles instead of zeros.

ii) $s=0$ with multiplicity $\kappa_{-}-\kappa_{0}$. If $\kappa_{-}-\kappa_{0}<0, s=0$ is a pole. Note that the contributions form the zeros (poles) from $\Delta(s)$ and the poles (zeros) from the summation over $j$ and $\rho$, respectively, cancel each other.

B) The Selberg super-zeta function $R_{1}(s)$ has "non-trivial" zeros and poles at the following points and nowhere else [27]:

i) $s=i p_{n}^{B(F)}$ : there are zeros (poles) of the same multiplicity as the corresponding eigenvalue of $\square$.

ii) $s=-i p_{n}^{B(F)}:$ reversed situation for poles and zeros.

iii) $s=\lambda_{n}^{B(F)}-\frac{1}{2}$ there are zeros (poles), and

iv) $s=-\left(\lambda_{n}^{F}-\frac{1}{2}\right)$ there are poles of the same multiplicity as the corresponding Eigenvalues of $\square$, respectively. The last two cases describe so-called small eigenvalues of the operator $\square$. All these "nontrivial" eigenvalues are supernumbers $s \in \Lambda_{\infty}$.

Of course, Eq. (5.17) can be extended meromorphically to all $s \in \Lambda_{\infty}$.

The test functions $h_{1}\left(i p+\frac{1}{2}, s, a\right)$ is symmetric by the interchange $s \rightarrow-s$. Therefore subtracting the trace formula for $h_{1}\left(i p+\frac{1}{2}, s, a\right)$ and $h_{1}\left(i p+\frac{1}{2},-s, a\right)$ yields the functional equation for $R_{1}$ in differential form

$$
\begin{aligned}
& \frac{d}{d s} \ln \left[R_{1}(s) R_{1}(-s)\right]=-A \operatorname{dim} V \tan \pi s+\frac{2\left(\kappa_{-}-\kappa_{0}\right)}{s} \\
& \quad-2 \sum_{\{R\}} \sum_{k=1}^{v-1} \frac{\operatorname{str}\left[U^{k}(R)\right]}{v} \chi_{R}^{k} \sum_{l=0}^{\infty} \cos \left[(2 l+1) \frac{k \pi}{v}\right]\left(\frac{1}{s+l+\frac{1}{2}}+\frac{1}{s-\left(l+\frac{1}{2}\right)}\right)
\end{aligned}
$$

(note $\left.\Psi\left(\frac{1}{2}+s\right)=\Psi\left(\frac{1}{2}-s\right)+\pi \tan \pi s\right)$. The integrated functional equation therefore has the form

$$
R_{1}(s) R_{1}(-s)=\text { const. }(\cos \pi s)^{A \operatorname{dim} V / \pi} s^{2\left(\kappa--\kappa_{0}\right)} \Psi_{1}(s),
$$

with the function $\Psi_{1}(s)$ given by

$$
\begin{aligned}
\Psi_{1}(s)= & \exp \left\{-2 \sum_{\{R\}} \sum_{k=1}^{v-1} \frac{\operatorname{str}\left[U^{k}(R)\right]}{v} \chi_{R}^{k}\right. \\
& \left.\times \sum_{l=0}^{\infty} \cos \left[(2 l+1) \frac{k \pi}{v}\right] \ln \left|s^{2}-\left(l+\frac{1}{2}\right)^{2}\right|\right\} .
\end{aligned}
$$


We check easily the consistence of the functional equation with respect to the analytical properties of the Selberg super-zeta function $R_{1}$.

2. The Selberg super-zeta function $R_{0}$. Let us turn to the discussion of the Selberg super-zeta function $R_{0}$. We consider the testfunction $(\mathfrak{R}(s, a)>1)$

$$
h_{0}\left(i p+\frac{1}{2}, s, a\right)=2\left(\frac{1}{2}+i p\right)\left(\frac{1}{s^{2}-\left(\frac{1}{2}+i p\right)^{2}}-\frac{1}{a^{2}-\left(\frac{1}{2}+i p\right)^{2}}\right),
$$

with the Fourier transform $g_{0}(u, s, a)$ given by

$$
g_{0}(u, s, a)=\operatorname{sign}(u) e^{u / 2}\left(e^{-s|u|}-e^{-a|u|}\right) .
$$

Again a regularization term is needed to match the requirements of a valid test function for the trace formula. Similarly as for $R_{1}$ we obtain the Selberg super trace formula for the test function $h_{0}\left(i p+\frac{1}{2}, s, a\right)$ as follows:

$$
\begin{aligned}
\frac{R_{0}^{\prime}(s)}{R_{0}(s)}- & \frac{R_{0}^{\prime}(a)}{R_{0}(a)} \\
= & 2 \sum_{n=1}^{\infty}\left[\frac{\lambda_{n}^{B}}{s^{2}-\left(\lambda_{n}^{B}\right)^{2}}-\frac{\lambda_{n}^{B}}{a^{2}-\left(\lambda_{n}^{B}\right)^{2}}-\frac{\lambda_{n}^{F}}{s^{2}-\left(\lambda_{n}^{F}\right)^{2}}+\frac{\lambda_{n}^{F}}{a^{2}-\left(\lambda_{n}^{F}\right)^{2}}\right] \\
& -\sum_{\{R\}} \sum_{k=1}^{v-1} \frac{\operatorname{str}\left[U^{k}(R)\right]}{v \sin (2 k \pi / v)} \\
& \times \sum_{l=1}^{\infty} \sin \left(\frac{2 l k \pi}{v}\right)\left[\frac{1}{s+l-1}-\frac{1}{s+l+1}-\frac{1}{a+l-1}+\frac{1}{a+l+1}\right] \\
& -\frac{A \operatorname{dim} V}{2 \pi}[\Psi(s)+\Psi(s+1)-\Psi(a)-\Psi(a+1)] \\
& -\frac{\kappa_{0}}{2}\left(\frac{1}{s-\frac{1}{2}}+\frac{1}{s+\frac{1}{2}}-\frac{1}{a-\frac{1}{2}}-\frac{1}{a+\frac{1}{2}}\right) \\
+ & \frac{1}{2} \operatorname{tr}\left[\mathscr{S}\left(\frac{1}{2}\right)\right]\left(\frac{1}{s^{2}-\frac{1}{4}}-\frac{1}{a^{2}-\frac{1}{4}}\right)+\kappa_{-} \frac{\Delta^{\prime}(s+1)}{\Delta(s+1)}-\kappa_{-} \frac{\Delta^{\prime}(a+1)}{\Delta(a+1)} \\
+ & \kappa_{-} \sum_{j=1}^{M}\left[\frac{1}{s+\left(\sigma_{j}-1\right)}-\frac{1}{s-\left(\sigma_{j}-1\right)}-\frac{1}{a+\left(\sigma_{j}-1\right)}+\frac{1}{a-\left(\sigma_{j}-1\right)}\right] \\
& \left.\frac{1}{s-\rho}-\frac{1}{s+\rho}-\frac{1}{a-\rho}\right]
\end{aligned}
$$

From Eq. (5.24) we read off

Theorem 5.2. The Selberg super-zeta function $R_{0}(s)$ is a meromorphic function on $\Lambda_{\infty}$ and has furthermore the following properties: 
A) The Selberg super-zeta function $R_{0}(s)$ has "trivial" zeros at the following points and nowhere else:

i) First note that

$$
\begin{gathered}
\frac{1}{\sin (2 k \pi / v)} \sum_{l=1}^{\infty} \sin \left(\frac{2 l k \pi}{v}\right)\left(\frac{1}{s+l-1}-\frac{1}{s+l+1}\right) \\
=\frac{1}{s}+\frac{\cos \left(\frac{2 k \pi}{v}\right)}{s+1}+2 \sum_{l=2}^{\infty} \frac{\cos \left(\frac{2 l k \pi}{v}\right)}{s+l} .
\end{gathered}
$$

Therefore:

$s=0$ with multiplicity

$$
\# N_{0}=\frac{A \operatorname{dim} V}{2 \pi}-\sum_{\{R\}} \sum_{k=1}^{v-1} \frac{\operatorname{str}\left[U^{k}(R)\right]}{v},
$$

$s=-1$ with multiplicity

$$
\# N_{1}=\frac{A \operatorname{dim} V}{\pi}-2 \sum_{\{R\}} \sum_{k=1}^{v-1} \frac{\operatorname{str}\left[U^{k}(R)\right]}{v} \cos \left(\frac{2 k \pi}{v}\right),
$$

$s=-n(n=2,3, \ldots)$ with multiplicity

$$
\# N_{n}=\frac{A \operatorname{dim} V}{\pi}-2 \sum_{\{R\}} \sum_{k=2}^{v-1} \frac{\operatorname{str}\left[U^{k}(R)\right]}{v} \sum_{l=2}^{\infty} \cos \left(\frac{2 l k \pi}{v}\right) .
$$

Note that if \# $N_{n}<0$, we have poles instead of zeros.

ii) $s=\frac{1}{2}$ with multiplicity \# $N_{\frac{1}{2}}=\kappa_{-} \operatorname{tr}\left[\mathscr{S}\left(\frac{1}{2}\right)\right]$.

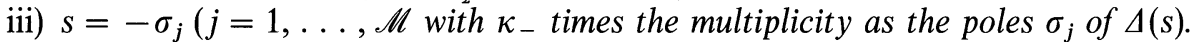

iv) $s=\rho$ with $\kappa_{-}$times the multiplicity as the pole $\rho$ of $\Delta(s)$ in the half-plane $\mathfrak{R}(s)<\frac{1}{2}$.

B) The Selberg super-zeta function $R_{0}(s)$ has "trivial" poles at the following points and nowhere else:

i) $s= \pm \frac{1}{2}$ with multiplicity $\kappa_{0} / 2$.

ii) $s=-\frac{1}{2}$ with multiplicity \# $N_{-\frac{1}{2}}=\kappa_{-} \operatorname{tr}\left[\mathscr{S}\left(\frac{1}{2}\right)\right]$.

iii) $s=1-\sigma_{j}(j=1, \ldots, \mathscr{M})$ with $\kappa_{-}$times the multiplicity of the pole $\sigma_{j}$ of the function $\Delta(s)$.

iv) $s=\rho-1$ with $\kappa_{-}$times the multiplicity as the pole $\rho$ of $\Delta(s)$ in the half-plane $\mathfrak{R}(s)<\frac{1}{2}$.

The items Aii)-Av) and Biii)-v) are only present if $\kappa_{0} \neq 0$.

C) The Selberg super-zeta $R_{0}(s)$ has "non-trivial" zeros and poles at the following points and nowhere else [27]:

i) $s=i p_{n}^{B(F)}-\frac{1}{2}$ : there are zeros (poles) of the same multiplicity as the corresponding Eigenvalue of $\square$.

ii) $s=-i p_{n}^{B(F)}-\frac{1}{2}$ : reversed situation for poles and zeros.

iii) $s=\lambda_{n}^{B(F)}$ there are zeros (poles), and

iv) $s=-\lambda_{n}^{B(F)}$ there are poles of the same multiplicity as the corresponding eigenvalue of $\square$, respectively. The last two cases describe so-called small eigenvalues of the operator $\square$. All these "nontrivial" eigenvalues are supernumbers $s \in \Lambda_{\infty}$. 
Of course, Eq. (5.24) can be extended meromorphically to all $s \in \Lambda_{\infty}$.

The test function $h_{0}\left(i p+\frac{1}{2}, s, a\right)$ is symmetric with respect to $s \rightarrow-s$. Therefore subtracting the trace formulae of $h_{0}\left(i p+\frac{1}{2}, s, a\right)$ and $h_{0}\left(i p+\frac{1}{2},-s, a\right)$ from each other yields the functional equation for the $R_{0}$-function in differential form

$$
\begin{aligned}
& \frac{d}{d s} \ln \left[R_{0}(s) R_{0}(-s)\right] \\
& =\frac{A \operatorname{dim} V}{\pi} \frac{d}{d s} \ln (\sin \pi s)+\left[\frac{\Delta^{\prime}(s)}{\Delta(s)}-\frac{\Delta^{\prime}(1+s)}{\Delta(1+s)}\right]^{\kappa-}+\kappa_{0}\left(\frac{1}{s-\frac{1}{2}}+\frac{1}{s+\frac{1}{2}}\right) \\
& \quad-\sum_{\{R\}} \sum_{k=1}^{v-1} \frac{\operatorname{str}\left[U^{k}(R)\right]}{v \sin (2 k \pi / v)} \\
& \quad \times \sum_{l=1}^{\infty} \sin \left(\frac{2 l k \pi}{v}\right)\left[\frac{1}{s+l-1}+\frac{1}{s-(l-1)}-\frac{1}{s+l+1}-\frac{1}{s-(l+1)}\right] .
\end{aligned}
$$

In integrated form, this gives the functional equation

$$
R_{0}(s) R_{0}(-s)=\text { const. }(\sin \pi s)^{A \operatorname{dim} V / \pi}\left(\frac{\Delta(s+1)}{\Delta(s)}\right)^{\kappa_{-}} \ln \left(s^{2}-\frac{1}{4}\right)^{-\kappa_{0}} \Psi_{0}(s),
$$

with the function $\Psi_{0}(s)$ given by

$$
\Psi_{0}(s)=\exp \left\{-\sum_{\{R\}} \sum_{k=1}^{v-1} \frac{\operatorname{str}\left[U^{k}(R)\right]}{v \sin (2 k \pi / v)} \sum_{l=1}^{\infty} \sin \left(\frac{2 l k \pi}{v}\right) \ln \left|\frac{\left(s^{2}-(l-1)^{2}\right)}{\left(s^{2}-(l+1)^{2}\right)}\right|\right\} .
$$

We check easily the consistence of the functional equation with respect to the analytical properties of the Selberg super-zeta function $R_{0}$. Note the similarity of the corresponding relation (5.4) for the classical Selberg zetafunction.

3. The Selberg super-zeta function $Z_{S}$. Following Ref. [42] we can also introduce the Selberg super-zeta function $Z_{S}(s)$ defined by

$$
Z_{S}(s)=\frac{Z_{0}(s) Z_{0}(s+1)}{Z_{1}^{2}\left(s+\frac{1}{2}\right)}
$$

The appropriate test function is $(\mathfrak{R}(s)>1)$

$$
h_{S}(p, s)=\left.\frac{1}{s^{2}-\lambda^{2}}\right|_{\lambda=\frac{1}{2}+i p}=\frac{1}{\left(s^{2}-\frac{1}{4}\right)-i p+p^{2}} .
$$

The corresponding Fourier transform $g_{S}$ is given by

$$
g_{S}(u, s)=\frac{1}{2 s} e^{u / 2-s|u|}
$$


The evaluation of the various terms in the Selberg super-trace formula is straightforward and we obtain similarly to the previous two cases

$$
\begin{aligned}
\frac{1}{2 s} \frac{Z_{S}^{\prime}(s)}{Z_{S}(s)}=\sum_{n=1}^{\infty} & {\left[\frac{1}{s^{2}-\left(\lambda_{n}^{B}\right)^{2}}-\frac{1}{s^{2}-\left(\lambda_{n}^{F}\right)^{2}}\right]+\left(\Delta_{0}^{(0)}+\frac{A \operatorname{dim} V}{4 \pi}\right) \frac{1}{s^{2}} } \\
& -\frac{1}{2 s} \sum_{\{R\}} \sum_{k=1}^{v-1} \frac{\operatorname{str}\left[U^{k}(R)\right]}{v \sin (2 k \pi / v)} \sum_{l=1}^{\infty} \sin \left(\frac{2 l k \pi}{v}\right) \\
& \times\left[\frac{4\left(1-\chi_{R}^{k} \cos \left(\frac{k \pi}{v}\right)\right)}{s+l}+\frac{1}{s+l-1}+\frac{1}{s+l+1}-\frac{2}{s+l}\right] \\
& +\frac{1}{s}\left[\tilde{\kappa}_{0}+\kappa_{-} \ln |\operatorname{sedt}(1-U(S))|\right]+\frac{\kappa_{-}}{2} \frac{\operatorname{tr}\left[\mathscr{S}\left(\frac{1}{2}\right)\right]}{s^{2}-\frac{1}{4}} \\
& -\frac{\kappa_{-}}{2 s} \frac{1}{s+\frac{1}{2}+\frac{\kappa_{0}}{4 s}\left(\frac{1}{s+\frac{1}{2}}-\frac{1}{s-\frac{1}{2}}\right)} \\
& +\frac{\tilde{\kappa}_{0}}{2 s}\left[\Psi\left(s+\frac{1}{2}\right)+\Psi\left(s+\frac{3}{2}\right)\right]-\frac{\kappa_{-}}{2 s} \frac{\Delta^{\prime}(1+s)}{\Delta(1+s)} \\
& -\kappa_{-} \sum_{j=1}^{M} \frac{1}{s^{2}-\left(\sigma_{j}-1\right)^{2}}+\kappa_{-} \sum_{\rho, \beta<\frac{1}{2}} \frac{1}{s^{2}-\rho^{2}} .
\end{aligned}
$$

From Eq. (5.34) we read off

Theorem 5.3. The Selberg super-zeta function $Z_{S}$ is a meromorphic function on $\Lambda_{\infty}$ and has furthermore the following properties:

A) The Selberg super-zeta function $Z_{S}(s)$ has "trivial" zeros at the following points and nowhere else:

i) $s=0$ with multiplicity

$$
\# N_{0}=2\left(\Delta_{0}^{(0)}+\frac{A \operatorname{dim} V}{4 \pi}\right)-\sum_{\{R\}} \sum_{k=1}^{v-1} \frac{\operatorname{str}\left[U^{k}(R)\right]}{v} .
$$

$s=-1$ with multiplicity

$$
\# N_{1}=-2 \sum_{\{R\}} \sum_{k=1}^{v-1} \frac{\operatorname{str}\left[U^{k}(R)\right]}{v}\left[1-2 \chi_{R} \cos \left(\frac{k \pi}{v}\right)+\cos \left(\frac{k \pi}{v}\right)\right] .
$$

$s=-n(n=-2,3,4, \ldots)$ with multiplicity

$$
\begin{aligned}
\# N_{n}= & 4 \sum_{\{R\}} \sum_{k=2}^{v-1} \frac{\operatorname{str}\left[U^{k}(R)\right]}{v \sin (2 k \pi / v)} \\
& \times\left[\sin ^{2}\left(\frac{k \pi}{v}\right)-\left(1-\chi_{R} \cos \frac{k \pi}{v}\right)\right] \sin \left(\frac{2 l k \pi}{v}\right) .
\end{aligned}
$$

ii) $s= \pm \frac{1}{2}$ with multiplicity $\# N_{ \pm \frac{1}{2}}=\kappa_{-} \operatorname{tr}\left[\mathscr{S}\left(\frac{1}{2}\right)\right] / 2$.

iii) $s=\rho$ with $\kappa_{\text {-times }}$ the multiplicity as the pole $\rho$ of $\Delta(s)$ in the half-plane $\mathfrak{R}(s)<\frac{1}{2}$.

iv) $s=\rho-1$ with $\kappa_{-}$times the multiplicity as the pole $\rho$ of $\Delta(s)$ in the half-plane $\mathfrak{R}(s)<\frac{1}{2}$. 
B) The Selberg super-zeta function $Z_{S}(s)$ has "trivial" poles at the following points and nowhere else:

i) $s=\frac{1}{2}$ with multiplicity $\kappa_{0} / 2$.

ii) $s=-\frac{1}{2}$ with multiplicity $2 \kappa_{-}-\kappa_{0} / 2$.

iii) $s=-\frac{1}{2}-l(l=1,2, \ldots)$ with multiplicity \# $N_{l}=2 \tilde{\kappa}_{0}$.

iv) $s=-\sigma_{j}(j=1, \ldots, \mathscr{M})$ with $\kappa_{-}$the multiplicity of the pole $\sigma_{j}$ of the function $\Delta(s)$.

The items Aii)-Aiv) and $\mathrm{Bii})-\mathrm{Bv}$ ) are only present if $\kappa_{-} \neq 0$.

C) The Selberg super-zeta function $Z_{S}(s)$ has "non-trivial" zeros and poles at the following points and nowhere else [27, 42]:

i) $s= \pm\left(\frac{1}{2}+i p_{n}^{B}\right)$ there are zeros (poles) and

ii) $s= \pm\left(\frac{1}{2}+i p_{n}^{F}\right)$ there are poles (zeros),

with the same multiplicity as the corresponding eigenvalue of $\square$, respectively.

Of course, Eq. (5.34) can be extended meromorphically to all $s \in \Lambda_{\infty}$.

The test function $h_{S}\left(i p+\frac{1}{2}, s\right)$ is symmetric with respect to $s \rightarrow-s$ and therefore we can deduce the functional relation

$$
\begin{aligned}
\frac{Z_{S}(s)}{Z_{S}(-s)}= & \text { const. } e^{4\left[\kappa_{0}+\kappa_{-} \ln |\operatorname{sdet}(1 U(S))|\right]} \\
& \times\left(\frac{1}{\Delta(s) \Delta(s+1)}\right)^{\kappa_{-}}\left(\frac{s-\frac{1}{2}}{s+\frac{1}{2}}\right)^{\kappa_{-}-\kappa_{0}}\left(\frac{\Gamma\left(s+\frac{1}{2}\right) \Gamma\left(s+\frac{3}{2}\right)}{\Gamma\left(\frac{1}{2}-s\right) \Gamma\left(\frac{3}{2}-s\right)}\right)^{\tilde{\kappa}_{0}} \Psi_{S}(s),
\end{aligned}
$$

with the function $\Psi_{S}(s)$ given by

$$
\begin{aligned}
\Psi_{S}(s)= & \exp \left\{-2 \sum_{\{R\}} \sum_{k=1}^{v-1} \frac{\operatorname{str}\left[U^{k}(R)\right]}{v \sin (2 k \pi / v)} \sum_{l=1}^{\infty} \sin \left(\frac{2 l k \pi}{v}\right)\right. \\
& \left.\times\left[2\left(1-2 \chi_{R}^{k} \cos \frac{k \pi}{v}\right) \ln \left|\frac{s+l}{s-l}\right|+\ln \left|\frac{(s+l-1)(s+l+1)}{(s-l+1)(s-l-1)}\right|\right]\right\} .
\end{aligned}
$$

We check easily the consistence of the functional equation with respect to the analytical properties of the Selberg super-zeta function $Z_{S}$. In the case, where only hyperbolic conjugacy classes are present in the super Fuchsian group, Eq. (5.38) reduces to the simple functional equation [27]

$$
Z_{S}(s)=Z_{S}(-s) \text {. }
$$

Let us note that the relation

$$
\begin{aligned}
\frac{d}{d s} \ln \left[\frac{Z_{0}(s) Z_{0}(s+1)}{Z_{1}\left(s+\frac{1}{2}\right)}\right] & -\frac{d}{d s} \ln \left[\frac{Z_{0}(s+1) Z_{0}(s+2)}{Z_{1}\left(s+\frac{3}{2}\right)}\right] \\
& =\frac{R_{0}^{\prime}(s)}{R_{0}(s)}+\frac{R_{0}^{\prime}(s+1)}{R_{0}(s+1)}-2 \frac{R_{1}^{\prime}\left(s+\frac{1}{2}\right)}{R_{1}\left(s+\frac{1}{2}\right)}
\end{aligned}
$$


provides a consistency check for the zeta functions $R_{0}, R_{1}$ and $Z_{S}$, respectively. This concludes the discussion.

\section{Summary and Discussion}

In this paper I have discussed a super extension of the Selberg trace formula, the Selberg super-trace formula, incorporating elliptic and parabolic conjugacy classes. Whereas the incorporation of the elliptic conjugacy classes were straightforward, I had to regularize the case of the incorporation of parabolic conjugacy classes by appropriate super Eisenstein series representing the continuous spectrum of the Dirac-Laplace operator $\square$ on the super Riemann surface. I obtained a closed version of all the relevant contributions.

Furthermore, I could discuss Selberg super-zeta functions. In this discussion similarly as in the classical case, there appeared additional "trivial zeros" and "trivial poles" in comparison to the "trivial zeros" of the super-zeta function due to the additional elliptic and parabolic conjugacy classes. In particular, the elliptic conjugacy classes only altered the multiplicity of the trivial poles already due to the hyperbolic conjugacy classes, the parabolic terms introduced new structure (zeros and poles) due to the poles $\sigma_{j}$ and $\rho=\beta+i \gamma$, respectively, of the function $\Delta(s)$. For the $R_{1}$-function only a zero for $s=0$ was added, and remained quite regular. Quite crucially it turned out that the dependence on the spin structure of the super Riemann surface, in particular if the corresponding $\chi_{s}$ for a parabolic element equals one, the trace formula and analytical structure of the Selberg zeta functions is far more regular than in the classical case [64]. For the character $\chi_{s}=1$ corresponding to a parabolic conjugacy class all the additional structure is smoothed out into a quite regular behaviour. The "nontrivial zeros" and "nontrivial poles" corresponding to the even and odd eigenvalues of the Dirac-Laplace operator were, of course, the same as in a previous publication [27]. Also functional relations could be derived for the Selberg super-zeta functions.

The question remains how to calculate superdeterminants of the operators $\square_{m}$ in the case of super-Riemann surfaces in the compact, respectively the noncompact case. A direct evaluation along the lines of Refs. $[1,27]$ is not obvious and, more important, it is not clear for general non-cocompact (super) Fuchsian group whether the operators $\Delta_{m}$, respectively $\square_{m}$, have infinitely many eigenvalues (compare [64]). It is however tempting to follow the approach of Ref. [59] and define them by means of the results of Refs. [1, 7, 27]. That is, one could propose

$$
\operatorname{sdet}\left(-\square_{m}\right) \propto \begin{cases}\frac{Z_{0}\left(1+\frac{m}{2}\right)}{Z_{1}\left(\frac{1+m}{2}\right)} & (m \text { even }) \\ Z_{1}\left(1+\frac{m}{2}\right) & (m \text { odd }),\end{cases}
$$


and similarly for negative even and odd $m$, respectively. A closer analysis of this problem is, however, beyond the scope of this paper and devoted to future investigations.

Acknowledgement. This work was supported by Deutsche Forschungsgemeinschaft under contract no. DFG Gr 1031. Furthermore I would like to thank the organizers of the symposium "Hyperbolic Spaces and Mathematical Physics" in Bielefeld (Germany), S. Albeverio, H. Helling, J.L. Mennicke and A.B. Venkov for their invitation and fruitful discussions, in particular with J. Bolte, H. Ninnemann and F. Steiner.

\section{References}

1. Aoki, K.: Heat Kernels and Super Determinants of Laplace Operators on Super Riemann Surfaces. Commun. Math. Phys. 117, 405-429 (1988)

2. Arthur, J.: The Selberg Trace Formula for Groups of F-Rank One. Ann. Math. 100, 326-385 (1974)

3. Aurich, R., Bogomolny, E.B., Steiner, F.: Periodic Orbits on the Regular Hyperbolic Octagon. Physica D48, 91-101 (1991); Aurich, R., Sieber, M., Steiner, F.: Quantum Chaos of the Hadamard-Gutzwiller Model. Phys. Rev. Lett. 61, 483-487 (1988)

4. Aurich, A., Steiner, F.: On the Periodic Orbits for a Strongly Chaotic System. Physica D32, 451-460 (1988); Periodic-Orbit Sum Rules for the Hadamard-Gutzwiller Model. Physica D39, 169-193 (1989); Energy-Level Statistics of the Hadamard-Gutzwiller Ensemble. Physica D43, 155-180 (1990); Exact Theory for the Quantum Eigenstates of the HadamardGutzwiller Model. Physica D48, 445-470 (1991); From Classical Periodic Orbits to the Quantization of Chaos. Proc. Roy. Soc. London A437, 693-719 (1992)

5. Balazs, N.L., Voros, A.: Chaos on the Pseudosphere. Phys. Rep. 143, 109-240 (1986)

6. Baranov, A.M., Manin, Yu.I., Frolov, I.V., Schwarz, A.S.: The Multiloop Contribution in the Fermionic String. Sov. J. Nucl. Phys. 43, 670-671 (1986)

7. Baranov, A.M., Manin, Yu.I., Frolov, I.V., Schwarz, A.S.: A Superanalog of the Selberg Trace Formula and Multiloop Contributions for Fermionic Strings. Commun. Math. Phys. 111, 373-392 (1987)

8. Baranov, A.M., Frolov, I.V., Shvarts, A.S.: Geometry of Two-Dimensional Superconformal Field Theories. Theor. Math. Phys. 70, 64-72 (1987)

9. Baranov, A.M., Schwarz, A.S.: Multiloop Contribution to String Theory. JETP Lett. 42, 419-421 (1985); On the Multiloop Contributions to the String Theory. Int. J. Mod. Phys. A2, 1773-1796 (1987)

10. Batchelor, M.: The Structure of Supermanifolds. Trans. Am. Math. Soc. 253, 329-338 (1979); Two Approaches to Supermanifolds. Trans. Am. Math. Soc. 258, 257-270 (1980)

11. Batchelor, M., Bryant, P.: Graded Riemann Surfaces. Commun. Math. Phys. 114, 243-255 (1988)

12. Bolte, J., Steiner, F.: Determinants of Laplace-like Operators on Riemann Surfaces. Commun. Math. Phys. 130, 581-597 (1990)

13. Bolte, J., Steiner, F.: The Selberg Trace Formula for Bordered Riemann Surfaces. DESY preprint, DESY 90-082, July 1990, 1-14

14. DeWitt, B.: Supermanifolds. Cambridge: Cambridge University Press 1984

15. D'Hoker, E., Phong, D.H.: Loop Amplitudes for the Bosonic Polyakov String. Nucl. Phys. B269, 205-234 (1986)

16. D'Hoker, E., Phong, D.H.: On Determinants of Laplacians on Riemann Surfaces. Commun. Math. Phys. 104, 537-545 (1986)

17. D'Hoker, E., Phong, D.H.: Loop Amplitudes for the Fermionic String. Nucl. Phys. B278, 225-241 (1986)

18. D'Hoker, E., Phong, D.H.: The Geometry of String Perturbation Theory. Rev. Mod. Phys. 60, 917-1065 (1988)

19. Erdélyi, A., Magnus, W., Oberhettinger, F., Tricomi, F.G. (eds.): Tables of Integral Transforms, Vol. I\&II. New York: McGraw Hill 1954

20. Gliozzi, F., Scherk, J., Olive, D.: Supergravity and the Spinor Dual Model. Phys. Lett. B65, 282-286 (1976); Supersymmetry, Supergravity Theories and the Spinor Dual Model. Nucl. Phys. B122, 253-290 (1977) 
21. Gradshteyn, I.S., Ryzhik, I.M.: Table of Integrals, Series, and Products. New York: Academic Press 1980

22. Green, M.B.: Supersymmetrical Dual String Theories and Their Field Theory Limits. Surveys in High Energy Physics 3, 127-160 (1983)

23. Green, M.B., Schwarz, J.H.: Supersymmetrical Dual String Theory, I-III. Nucl. Phys. B181, 502-530 (1982), B198, 252-268, 441-460 (1982)

24. Green, M.B., Schwarz, J.H.: Anomaly Cancellations in Supersymmetric $D=10$ Gauge Theory and Superstring Theory. Phys. Lett. B149, 117-122 (1984); Infinity Cancellations in SO(32) Superstring Theory. Phys. Lett. B151, 21-25 (1985); The Hexagon Gauge Anomaly in Type I Superstring Theory. Nucl. Phys. B255, 93-114 (1985)

25. Green, M.B., Schwarz, J.H., Witten, E.: Superstring Theory I\&II. Cambridge: Cambridge University Press 1988

26. Grosche, C.: The Path Integral on the Poincaré Upper Half-Plane With a Magnetic Field and for the Morse Potential. Ann. Phys. (N.Y.) 187, 110-134 (1988); The Path Integral on the Poincaré Disc, the Poincare Upper Half-Plane and on the Hyperbolic Strip. Fortschr. Phys. 38, 531-569 (1990); Path Integration on the Hyperbolic Plane With a Magnetic Field. Ann. Phys. (N.Y.) 201, 258-284 (1990); Path Integration on Hyperbolic Spaces. J. Phys. A.: Math. Gen. 25, 4211-4244 (1992)

27. Grosche, C.: Selberg Supertrace Formula for Super Riemann Surfaces, Analytic Properties of the Selberg Super-zeta-Functions and Multiloop Contributions to the Fermionic String. DESY Preprint DESY 89-010, February 1989, 1-102, and Commun. Math. Phys. 133, 433-485 (1990)

28. Grosche, C., Steiner, F.: The Path Integral on the Poincare Upper Half Plane and for Liouville Quantum Mechanics. Phys. Lett. A123, 319-328 (1987)

29. Gross, D.J., Periwal, V.: String Perturbation Theory Diverges. Phys. Rev. Lett. 60, 2105-2108 (1988)

30. Gutzwiller, M.C.: Phase-Integral Approximation in Momentum Space and the Bound States of an Atom. J. Math. Phys. 8, 1979-2000 (1967); Phase-Integral Approximation in Momentum Space and the Bound States of an Atom. II. J. Math. Phys. 10, 1004-1020 (1969); Energy Spectrum According to Classical Mechanics. J. Math. Phys. 11, 1791-1806 (1970); Periodic Orbits and Classical Quantization Conditions. J. Math. Phys. 12, 343-358 (1971).

31. Gutzwiller, M.C.: The Quantization of a Classically Ergodic System. Physica D5, 183-207 (1982); The Geometry of Quantum Chaos. Physica Scripta T9, 184-192 (1985); Physics and Selberg's Trace Formula. Contemp. Math. 53, 215-251 (1986); Mechanics on a Surface of Constant Curvature. In: Number Theory, Lect. Notes in Math., Vol. 1240, pp. 230-258. Berlin, Heidelberg, New York: Springer 1985

32. Hardy, G.H., Wright E.M.: The Theory of Numbers. Oxford: Oxford University Press 1960

33. Hejhal, D.A.: The Selberg Trace Formula and the Riemann Zeta Function. Duke Math. J. 43, 441-482 (1976)

34. Hejhal, D.A.: The Selberg Trace Formula for PSL(2, R), I\&II. Lect. Notes in Math. Vols. 548 \& 1001. Berlin, Heidelberg, New York: Springer 1976, 1981

35. Howe, P.S.: Superspace and the Spinning String. Phys. Lett. B70, 453-456 (1977); Super Weyl Transformations in Two Dimensions. J. Phys. A: Math. Gen. 12, 393-402 (1979)

36. Kubota, T.: Elementary Theory of Eisenstein Series Tokio. Kodansha 1973

37. Littlejohn, R.G.: Semiclassical Structure of Trace Formulas: J. Math. Phys. 31, 2952-2977 (1990)

38. McKean, H.P.: Selberg's Trace Formula as Applied to a Compact Riemann Surface. Commun. Pure Appl. Math. 25, 225-246 (1972)

39. Magnus, W., Oberhettinger, F., Soni, R.: Formulas and Theorems for the Special Functions of Mathematical Physics. Berlin, Heidelberg, New York: Springer 1966

40. Manin, Yu.I.: The Partition Function of the Polyakov String Can Be Expressed in Terms of Theta-Functions. Phys. Lett. B172, 184-185 (1986)

41. Matsumoto, S., Yasui, Y.: Chaos on the Super Riemann Surface. Prog. Theor. Phys. 79, 1022-1027 (1988)

42. Matsumoto, S., Uehara, S., Yasui, Y.: Hadamard Model on the Super Riemann Surface. Phys. Lett. A134, 81-86 (1988)

43. Matsumoto, S., Uehara, S., Yasui, Y.: A Superparticle on the Super Riemann Surface. J. Math. Phys. 31, 476-501 (1990) 
44. Matthies, C., Steiner, F.: Selberg's Zeta Function and the Quantization of Chaos. Phys. Rev. A44, R7877-R7880 (1991)

45. Moore, G., Nelson, P., Polchinski, J.: Strings and Supermoduli. Phys. Lett. B169, 47-53 (1986)

46. Neveu, A., Schwarz, J.H.: Quark Model of Dual Pions. Phys. Rev. D4, 1109-1111 (1971)

47. Ninnemann, H.: Holomorphe and Harmonische Formen auf Super-Riemannschen Flächen und ihre Anwendung auf den Fermionischen String. Diploma Thesis, Hamburg University 1989; Deformations of Super Riemann Surfaces. DESY preprint DESY 90-120, 1-23

48. Oshima, K.: Completeness Relations for Maass Laplacians and Heat Kernels on the Super Poincaré Upper Half-Plane. J. Math. Phys. 31, 3060-3063 (1990)

49. Polyakov, A.M.: Quantum Geometry of Bosonic Strings. Phys. Lett. B103, 207-210 (1981)

50. Polyakov, A.M.: Quantum Geometry of Fermionic Strings. Phys. Lett. B103, 211-213 (1981)

51. Rabin, J.M., Crane, L.: Global Properties of Supermanifolds. Commun. Math. Phys. 100, 141-160 (1985); How Different are the Supermanifolds of Rogers and DeWitt? Commun. Math. Phys. 102, 123-137 (1985); Super Riemann Surfaces: Uniformization and Teichmüller Theory. Commun. Math. Phys. 113, 601-623 (1988)

52. Ramond, P.: Dual Theory for Free Fermions. Phys. Rev. D3, 2415-2418 (1971)

53. Rogers, A.: A Global Theory of Supermanifolds. J. Math. Phys. 21, 1352-1364 (1980); On the Existence of Gloal Integral Forms on Supermanifolds. J. Math. Phys. 26, 2749-2753 (1985); Graded Manifolds, Supermanifolds and Infinite-Dimensional Grassmann Algebras. Commun. Math. Phys. 105, 375-384 (1986)

54. Schwarz, J.H.: Superstring Theory. Phys. Rep. 89, 223-322 (1982)

55. Selberg, A.: Harmonic Analysis and Discontinuous Groups in Weakly Symmetric Riemannian Spaces With Application to Dirichlet Series. J. Indian Math. Soc. 20, 47-87 (1956)

56. Sieber, M.: The Hyperbola Billiard: A Model for the Semiclassical Quantization of Chaotic Systems. DESY preprint, DESY 91-030, April 1991, 1-101; Sieber, M., Steiner, F.: Generalized Periodic-Orbit Sum Rules for Strongly Chaotic Systems; Phys. Lett A144, 159-163 (1990); Quantization of Chaos; Phys. Rev. Lett. 67, 1941-1944 (1991)

57. Steiner, F.: On Selberg's Zeta Function for Compact Riemann Surfaces. Phys. Lett. B188, 447-454 (1987); Quantum Chaos and Geometry. In: Mitter, H., Pittner, L. (eds.) Recent Developments in Mathematical Physics. 26. Internationale Universitäswochen, Schladming 1987, pp. 305-312. Berlin, Heidelberg, New York: Springer 1987

58. Subia, N.: Formule de Selberg et Formes D'Espace Hyperboliques Compactes. Analyse Harmonique sur les Groupes de Lie. Lect. Notes in Math., Vol. 497, pp. 674-700. Berli!n, Heidelberg, New York: Springer 1975

59. Takhtajan, L.A., Zograf, P.G.: A Local Index Theorem for Families of $\bar{\partial}$-Operators on Punctured Riemann Surfaces and a New Kähler Metric on Their Moduli Spaces. Commun. Math. Phys. 137, 399-426 (1991)

60. Uehara, S., Yasui, Y.: A Superparticle on the "Super" Poincaré Upper Half Plane. Phys. Lett. B202, 530-534 (1988; Super-Selberg's Trace Formula from the Chaotic Model. J. Math. Phys. 29, 2486-2490 (1988)

61. Venkov, A.B.: Expansion in Automorphic Eigenfunctions of the Laplace Operator and the Selberg Trace Formula in the Space $S O_{0}(n, 1) / S O(n)$. Soviet Math. Dokl. 12, 1363-1366(1971)

62. Venkov, A.B.: Expansion in Automorphic Eigenfunctions of the Laplace-Beltrami Operator in Classical Symmetric Spaces of Rank One, and the Selberg Trace Formula. Proc. Math. Inst. Steklov 125, 1-48 (1973)

63. Venkov, A.B.: Selberg's Trace Formula and Non-Euclidean Vibrations of an Infinite Membrane. Sov. Math. Dokl. 19, 708-712 (1979)

64. Venkov, A.B.: Spectral Theory of Automorphic Functions. Proc. Math. Inst. Steklov 153, 1-163 (1981)

65. Venkov, A.B.: On the Selberg Trace Formula for the Automorphic Schroedinger Operator. Institut des Hautes Etudes Scientific preprint, IHES/M/90/98, December 1990, 1-18

66. Weil, A.: Sur les 'Formules Explicites' de la Théorie des Nombres Premiere. Comm. Sém. Math. Lund (Medd. Lunds Univ. Mat. Sem., Suppl. 252-265) 1952

67. Bolte, J., Grosche, C.: Selberg Trace Formula for Bordered Riemann Surfaces: Hyperbolic, Elliptic and Parabolic Conjugacy Classes, and Determinants of Maass-Laplacians. DESYpreprint, DESY 92-118, 1-27, August 1992 
